\title{
The Fundamental Scaling Relations of Elliptical Galaxies
}

\section{Citation}

Robertson, Brant, Thomas J. Cox, Lars Hernquist, Marijn Franx, Philip F. Hopkins, Paul Martini, and Volker Springel. 2006. “The Fundamental Scaling Relations of Elliptical Galaxies." The Astrophysical Journal 641 (1): 21-40. https://doi.org/10.1086/500360.

\section{Permanent link}

http://nrs.harvard.edu/urn-3:HUL.InstRepos:41381705

\section{Terms of Use}

This article was downloaded from Harvard University's DASH repository, and is made available under the terms and conditions applicable to Other Posted Material, as set forth at http:// nrs.harvard.edu/urn-3:HUL.InstRepos:dash.current.terms-of-use\#LAA

\section{Share Your Story}

The Harvard community has made this article openly available.

Please share how this access benefits you. Submit a story.

Accessibility 
DRAFT VERSION OCTOBER 17, 2018

Preprint typeset using $\mathrm{LT}_{\mathrm{E}} \mathrm{X}$ style emulateapj v. 9/08/03

\title{
THE FUNDAMENTAL SCALING RELATIONS OF ELLIPTICAL GALAXIES
}

\author{
Brant ROBERTSON ${ }^{1,5}$, THOMAS J. COX ${ }^{1}$, LARS HERnQUist ${ }^{1}$, MARIJN FranX ${ }^{2}$, \\ PhILIP F. HOPKINS ${ }^{1}$, PAUL MARTINI ${ }^{3}$, VOLKER SPRINGEL ${ }^{4}$ \\ Draft version October 17, 2018
}

\begin{abstract}
We examine the fundamental scaling relations of elliptical galaxies formed through mergers. Using hundreds of simulations to judge the impact of progenitor galaxy properties on the properties of merger remnants, we find that gas dissipation provides an important contribution to tilt in the Fundamental Plane relation. Dissipationless mergers of disks produce remnants that occupy a plane similar to that delineated by the virial relation. As the gas content of progenitor disk galaxies is increased, the tilt of the resulting Fundamental Plane relation increases and the slope of the $R_{\mathrm{e}}-M_{\star}$ relation steepens. For gas fractions $f_{\text {gas }}>30 \%$, the simulated Fundamental Plane scalings $\left(R_{\mathrm{e}} \propto \sigma^{1.55} I_{\mathrm{e}}^{-0.82}\right)$ approach those observed in the $K$-band $\left(R_{\mathrm{e}} \propto \sigma^{1.53} I_{\mathrm{e}}^{-0.79}\right)$. The dissipationless merging of spheroidal galaxies and the re-merging of disk galaxy remnants roughly maintain the tilt of the Fundamental Plane occupied by the progenitor ellipticals, approximately independent of the orbital energy or angular momentum. Dry merging of spheroidal systems at redshifts $z<1$ is then expected to maintain the stellar-mass Fundamental Plane relations imprinted by gas-rich merging during the epoch of rapid spheroid and supermassive black hole growth at redshifts $z \approx 1-3$. In our simulations, feedback from supermassive black hole growth has only a minor influence on the stellar-mass scaling relations of spheroidal galaxies, but may play a role in maintaining the observed Fundamental Plane tilt at optical wavelengths by suppressing residual star formation in merger remnants.

We estimate that $\approx 40-100 \%$ of the Fundamental Plane tilt induced by structural properties, as opposed to stellar population effects, owes to trends in the central total-to-stellar mass ratio $M_{\text {total }} / M_{\star}$ produced by dissipation. Gas cooling allows for an increase in central stellar phase-space density relative to dissipationless mergers, thereby decreasing the central $M_{\text {total }} / M_{\star}$. Lower mass systems obtain greater phase-space densities than higher mass systems, producing a galaxy mass-dependent central $M_{\text {total }} / M_{\star}$ and a corresponding tilt in the Fundamental Plane. We account for these trends in the importance of dissipation with galaxy mass in terms of the inefficient cooling of collisionally heated gas in massive halos and dynamically varying gas consumption timescales in smaller systems.
\end{abstract}

Subject headings: galaxies: formation - galaxies: evolution

\section{INTRODUCTION}

Elliptical galaxies represent a fascinating combination of complexity and regularity. A leading theory for the origin of early-type galaxies is based on mergers of disk galaxies (Toomre \& Toomre 1972; Toomre 1977) and likely involves gas dissipation, star formation, and supermassive black hole feedback (Barnes 1992; Barnes \& Hernquist 1992, 1996; Mihos \& Hernquist 1994, 1996; Di Matteo et al. 2005) in addition to stellar dynamics. Despite their complex origins, early-type galaxies obey a regular set of scaling relations that connect their photometric and kinematic properties, most notably the relation between effective radius $R_{\mathrm{e}}$, central stellar velocity dispersion $\sigma$, and average central surface brightness $I_{\mathrm{e}}$ known as the Fundamental Plane (FP; Dressler et al. 1987; Diorgovski \& Davis 1987)

$$
R_{\mathrm{e}} \propto \sigma^{\alpha} I_{\mathrm{e}}^{-\beta}
$$

The virial theorem can be used to calculate this relation for homologous systems, which gives $\alpha=2, \beta=1$ (e.g.,

\footnotetext{
${ }^{1}$ Harvard-Smithsonian Center for Astrophysics, 60 Garden St., Cambridge, MA 02138, USA.

${ }^{2}$ Leiden Observatory, P.O. Box 9513, NL-2300 RA Leiden, Netherlands.

${ }^{3}$ The Ohio State University, Department of Astronomy, 140 West 18th Ave., Columbus, OH 43210, USA.

4 Max-Planck-Institut für Astrophysik, Karl-Schwarzschild-Straße 1, 85740 Garching bei München, Germany.

5 brobertson@cfa.harvard.edu
}

Faber et al. 1987, hereafter the "virial scaling").

While the observational determination of the FP was originally motivated as a precise distance indicator to improve upon the previously known luminosity $(L)$ - velocity dispersion $(\sigma)$ relation (Faber \& Jackson 1976), the importance of the FP scalings and its correspondingly small scatter for theories of elliptical galaxy formation was also realized. The first observationally determined FP scalings $(\alpha \sim 1.3-$ 1.4, $\beta \sim 0.8-0.9$, at optical wavelengths; Dressler et al. 1987; Diorgovski \& Davis 1987) differed from the virial scaling, indicating a "tilt" relative to the expectation for homologous systems. The FP tilt implied that the mass-to-light ratio $M / L$ likely varies as a function of galaxy mass or luminosity as

$$
\frac{M}{L} \propto L^{\gamma}
$$

within the range $\gamma \approx 1 / 5-1 / 4$. Faber et al. (1987) noted that deviations from the FP can be induced by $M / L$ variance owing to e.g. metallicity or age trends in stellar populations, dynamical or structural properties, and the relative distribution of dark and baryonic matter. In principle, each of these effects may also introduce a systematic tilt into the observed FP if they vary as a function of elliptical galaxy mass.

The purpose of the current paper is to gauge the importance of various contributions to the tilt in the observed FP in the context of the scenario where elliptical galaxies form from mergers. Using hundreds of simulated galaxy mergers that include the physics of gas cooling, star formation, supernova 
feedback, and black hole accretion and feedback, we determine that gas dissipation may significantly contribute to the tilt of the observed FP, in addition to tilt induced by $M / L$ trends from stellar populations. We propose that elliptical galaxies initially form in gas-rich mergers from disk galaxy progenitors whose gas fractions exceed $f_{\text {gas }} \sim 30 \%$, and show that these remnants display substantial FP tilt.

We connect the origin of this tilt to the central stellar phasespace density of the remnants. In small mass systems where dissipation is most important, the stellar phase-space density of remnants increases substantially in high-gas fraction mergers. The central stellar phase-space density in massive ellipticals remains similar in mergers with varying gas fractions, with their stars on average obtaining lower phase-space densities than reached in smaller systems. This mass-dependent phase-space density trend translates into a mass-dependent trend in the ratio of total mass to stellar mass $M_{\text {total }} / M_{\star}$ in the central regions of ellipticals and a corresponding tilt in the FP. We then explain this mass-dependent importance of dissipation in terms of the inefficient cooling of collisionally heated gas in massive ellipticals and the dynamically varying gas consumption timescale of smaller systems. It is interesting, and possibly significant, that the gas fraction required to reproduce the observed tilt in the FP is, as discussed by Hernquist (1993) similar to that needed for mergers of disks to yield the high central phase space densities of ellipticals.

In accord with other work (e.g., Capelato et al. 1995; Dantas et al. 2003; Nipoti et al. 2003; Boylan-Kolchin et al. 2005), we find that subsequent dissipationless merging between spheroidal galaxies roughly maintains the FP tilt. Moreover, we find that a single generation of dissipationless re-merging of remnants will induce scatter in the $M_{\mathrm{BH}}-\sigma$ relation but will not destroy the correlation, as spheroidal galaxy mergers also do not dramatically alter the $R_{\mathrm{e}}-M_{\star}$ relation when viewed as a mass-sequence. Possible dry merging between spheroidal galaxies at redshifts $z<1$ as indicated by recent observations (Bell et al. 2005; van Dokkum 2005) is thus not expected to destroy tight FP or $M_{\mathrm{BH}}-\sigma$ relations generated during spheroid formation through gas-rich mergers at higher redshifts.

This paper is organized as follows. We review the observational and theoretical work on elliptical galaxy formation and scaling relations in $\$ 2$ We present our methodology in $\$ 3$ and our results in $\$ 4$. We discuss the implications of our work in $\$ 6$ and summarize and conclude in \$7 Throughout, we adopt a flat $\Lambda$ CDM universe with $\Omega_{\mathrm{M}}=0.3, \Omega_{\Lambda}=0.7, \Omega_{\mathrm{b}}=0.04$, and a Hubble parameter $H_{0}=100 h \mathrm{~km} \mathrm{~s}^{-1} \mathrm{Mpc}^{-1}$ with $h=0.7$.

\section{REVIEW OF ELLIPTICAL GALAXY SCALING LAWS}

Scaling laws describing the regularity of the properties of elliptical galaxies have been known since at least Fish (1964), who reported a relation between their potential energy $W$ and mass $M_{\star}$ as $W \propto M_{\star}^{3 / 2}$. Even earlier, de Vaucouleurs (1948, hereafter $\mathrm{dV}$ ) had demonstrated that ellipticals generally have a surface brightness profile $\log I(r) \propto r^{1 / 4}$, at least over some range in radius $r$, and implications of the $\mathrm{dV}$ profile for the mass-dependent properties of ellipticals were recognized before the Fish (1964) paper (e.g., Poveda 1958).

Sandage (1972) found a color-magnitude relation for Virgo and Coma cluster ellipticals (see also Stebbins \& Whitford 1952; de Vaucouleurs 1961). Faber (1973) discovered a similar color-magnitude trend in Local Group and cluster ellipticals, as well as an absorption-line strength-magnitude relation, and suggested that these properties of elliptical galax- ies depend primarily on galaxy luminosity. Faber \& Jackson (1976) established the relation between luminosity $L$ and velocity dispersion $\sigma$, providing further evidence that ellipticals follow a regular sequence as a function of mass. Kormendy (1977) showed that the surface brightnesses and effective radii of ellipticals correlate with galaxy luminosity and with one another (see also Binggeli et al. 1984).

Important early indications that a second parameter (in addition to mass) governs the properties of ellipticals came with the Terlevich et al. (1981) and Tonry \& Davis (1981) work that implied a correlation of $L-\sigma$ and absorption-line strength- luminosity relation $\left(\mathrm{Mg}_{2}-L\right)$ residuals. While these findings were later contradicted (e.g. Dressler 1984), that elliptical galaxies were not a one-parameter family remained an important possibility.

The discovery of the FP (Dressler et al. 1987; Diorgovski \& Davis 1987) definitively revealed that elliptical galaxy properties are set by at least two parameters. Specifically, ellipticals were found to obey a relation between $R_{\mathrm{e}}, \sigma$, and $I_{\mathrm{e}}$ as given by Equation (1), with less than half the scatter of the Faber \& Jackson (1976) $L-\sigma$ relation. The small scatter of the FP was immediately noticed, implying that the process of elliptical galaxy formation must result in a very regular mass-sequence. Faber et al. (1987) noted that mass-to-light ratio $(M / L)$ variations can influence observations of the FP by inducing tilt relative to the plane defined by the virial relation. Much of the subsequent work on the FP has centered around possible causes of $M / L$ variation or other origins for tilting the FP relative to the virial plane.

Numerous subsequent observations verified and improved the FP relation for ellipticals (Lucevet al. 1991a b; de Carvalho \& Diorgovski 1992; Bender et al. 1992; Jørgensen et al. 1992; Guzman et al. 1993; Jørgensen et al.|1993; Saglia et al.|1993; Bender et al.|1994; Prugniel \& Simien 1994; Pahre et al. 1995; Jørgensen et al. 1996; Prugniel \& Simien 1996; Busarello et al. 1997; Graham \& Colless 1997; Bender et al. 1998; Pahre et al. 1998b a; Mobasher et al. 1999; Kronawitter et al. 2000; Gerhard et al. 2001; Bernardi et al. 2003a; Padmanabhan et al. 2004; Woo et al. 2004; Cappellari et al. 2005) and extended the FP determinations to higher redshifts (Franx 1993; van Dokkum \& Franx 1996; Kelson et al. 1997; Schade et al. 1997; van Dokkum et al. 1998; Jørgensen et al. 1999; Treu et al. 1999; Kelson et al. 2000a b c; Kochanek et al. 2000; Treu et al. 2001; van Dokkum \& Franx 2001; van Dokkum et al. 2001; Treu et al. 2002; Gebhardt et al. 2003; Rusin et al. 2003; van Dokkum \& Stanford 2003; van Dokkum \& Ellis 2003; van der Wel et al. 2004). While the specific details may vary, these works generally conclude that

- A tight fundamental plane between the elliptical galaxy properties $R_{\mathrm{e}}, \sigma, I_{\mathrm{e}}$ exists, and extends in some form to at least redshift $z \sim 1$.

- Ellipticals are old, with formation redshifts $z>1$, and their color evolution, which controls the FP normalization, is roughly consistent with passive evolution of their stellar populations.

- Some of the FP tilt must originate from the change in stellar population $M / L$ with galaxy mass, but the extent to which stellar populations contribute to $M / L$-induced FP tilt is debated. 
Of specific interest to modelers are the observations that made definite statements about the influence of structural or kinematic nonhomology on $M / L$ and the FP tilt, especially those that conclude directly that these nonhomologies are either unimportant or of minor significance (Gerhard et al. 2001; Cappellari et al. 2005), significant (e.g. Padmanabhan et al. 2004), or of possible but as yet not fully determined importance (e.g. Pahre et al. 1998b).

Moreover, elliptical galaxy properties related to the FP or its projections have been extensively observed. These include studies of the photometric profiles (e.g. de Vaucouleurs 1948; Sersic 1968; Kormendy \& Illingworth 1982; Burkert 1993; Caon et al. 1993), internal kinematic structure (e.g. Binnev 1978; Davies et al. 1983; Davies \& Birkinshaw 1988; Bender et al. 1994), metallicity (de Carvalho \& Diorgovski 1992; Bender et al. 1993, 1996; Bernardi et al. 1998, 2003b), and the $M_{\mathrm{BH}}-\sigma$ relation (e.g. Gebhardt et al. 2000; Ferrarese \& Merritt 2000; Tremaine et al. 2002). An important, related property is the size-stellar mass relation (Shen et al. 2003; Truiillo et al. 2004: Truiillo \& Aguerri 2004; McIntosh et al. 2005), that indicates a power-law correlation between some characteristic galaxy size and the stellar mass or luminosity.

Interpreting these observations has been the focus of various theoretical efforts (see, e.g. Barnes \& Hernquist 1992, for a description of early results). Notably, simulations of the formation of ellipticals and their properties in the context of the merger hypothesis have been performed using a variety of progenitor models including spheroidal (White 1979; Capelato et al. 1995; Dantas et al. 2003; González-García \& van Albada 2003; Nipoti et al. 2003), disk (Toomre \& Toomre 1972; Gerhard 1981; Farouki \& Shapiro 1982; Farouki et al. 1983; Barnes \& Hernquist 1991; Barnes 1992; Hernquist 1992, 1993; Hernquist et al.|1993; Mihos \& Hernquist|1994, 1996; Hibbard \& van Gorkom 1996; Bekki| 1998; Dubinski |1998; Naab et al. 1999; Naab \& Burkert 2001; Aceves \& Velázquez 2005), and cosmological systems (e.g. Aarseth \& Fall 1980; Sáiz et al. 2004). Analytical models of elliptical galaxies have also been formulated (Hernquist 1990; Ciotti 1991; Ciotti et al. 1996; Ciotti 1996; Ciotti \& Bertin 1999), aiding the interpretation of both the observational and simulation results. In what follows, we combine features of many of these previous theoretical endeavors by simulating mergers between dissipational and dissipationless disk galaxies, spheroidal systems, and merger remnants to determine their fundamental scaling relations.

\section{METHODOLOGY}

Galaxy merging combines much complex physics, including e.g. the collisionless dynamics of dark matter and stars, gas dissipation, star formation, and feedback from supernovae and black hole growth. While each of these processes may in principle be essential for determining merger remnant properties, their relative importance has not been fully established. By comparing the structure of merger remnants in simulations which systematically include or exclude various processes, we attempt to both test the merger hypothesis and identify the most important physical mechanisms.

To this end, we perform a set of hundreds of simulations of galaxy mergers. Our suite consists of three categories of diskdisk mergers and one class of spheroid-spheroid mergers. The first category of simulations, referred to as "dissipationless," includes only simulations of disks consisting of collisionless stars and dark matter. The second category, termed "dissipational" mergers, consists of simulations that account for gas cooling, star formation, the physics of the interstellar medium, and supernova feedback. We refer to the third category of calculations as "full-model" simulations which, in addition to the processes accounted for in the "dissipational" mergers, also include supermassive black hole growth and feedback. All three categories of disk-disk mergers are derived from a standard set of galaxy models to enable a direct comparison of physical processes.

To perform our numerical simulations, we utilize the GADGET2 code (Springel 2005). GADGET2 uses the smoothed particle hydrodynamics (SPH) formalism (Lucy 1977; Gingold \& Monaghan 1977) in its entropy-conserving formulation (Springel \& Hernquist 2002) to calculate the dynamical evolution of the gas and a tree-based method to compute gravitational forces between particles (Barnes \& Hut 1986).

All progenitor galaxies were created using the methods described in Springel et al. (2005), which allow for the generation of stable equilibrium galaxy models. Each galaxy contains an extended dark matter halo, and may also consist of a stellar disk, gaseous disk, stellar bulge, and a supermassive black hole particle. The collisionless components of the galaxy models are required to satisfy the Jeans equations while the structure of the gas component is determined by the equation of hydrostatic equilibrium and an integral constraint on the surface mass density.

\subsection{Dissipationless Disk Simulations}

The dissipationless disk progenitors consist of an exponential stellar disk embedded in a dark matter halo with virial velocities in the range $V_{\text {vir }}=80-500 \mathrm{~km} \mathrm{~s}^{-1}$. We initialize the size of the disk according to the Mo et al. (1998) formalism for dissipational disk galaxy formation (see also Fall \& Efstathiou 1980; Blumenthal et al. 1986) assuming the disk contains a fraction of the total galaxy angular momentum equal to its mass fraction, which we set to $m_{\mathrm{d}} \equiv$ $M_{\text {disk }} / M_{\text {vir }}=0.041$ to match the Milky Way-like model used by Springel et al. (2005). The disk scalelength $r_{\mathrm{d}}$ is then determined by the galaxy spin $\lambda$ and the dark matter halo concentration $C_{\mathrm{vir}}$. We adopt $\lambda=0.033$, which is near the mode of the redshift- and mass- independent distribution of dark matter halo spins measured in cosmological N-body simulations (Vitvitska et al. 2002). For the Navarro-Frenk-White halo concentration $C_{\text {vir }}$ (Navarro et al. 1997, NFW), we adopt the mass- and redshift-dependent dark matter halo concentrations measured by Bullock et al. (2001)

$$
C_{\mathrm{vir}}\left(M_{\mathrm{vir}}, z\right) \approx 9\left(\frac{M_{\mathrm{vir}}}{M_{\mathrm{coll}, 0}}\right)^{-0.13}(1+z)^{-1},
$$

where $M_{\text {coll, } 0} \sim 8 \times 10^{12} h^{-1} M_{\odot}$ is the linear collapse mass at redshift $z=0$. In all cases, the stellar disk scaleheight $h_{\mathrm{d}}=$ $0.2 r_{\mathrm{d}}$, similar to the Milky Way (c.f. Siegel et al.|2002). We model the dark matter halo with a Hernquist (1990) density profile of the form

$$
\rho_{\mathrm{h}}(r)=\frac{M_{\mathrm{DM}}}{2 \pi} \frac{a}{r(r+a)^{3}},
$$

where the scalelength $a\left(C_{\text {vir }}\right)$ maps the Hernquist (1990) profile parameters to the appropriate NFW halo parameters (for details, see Springel et al. 2005). 
Table 1. Galaxy Mergers

\begin{tabular}{|c|c|c|c|c|c|c|}
\hline Model & $\begin{array}{c}\text { Progenitor } \\
V_{\text {vir }}\left[\mathrm{km} \mathrm{s}^{-1}\right]\end{array}$ & $\begin{array}{c}\text { Redshift } \\
z\end{array}$ & $\begin{array}{c}\text { Gas Fraction } \\
f_{\text {gas }}\end{array}$ & $\begin{array}{l}\text { ISM Pressurization } \\
q_{\text {EOS }}\end{array}$ & $\begin{array}{c}\text { Pericentric Separation } \\
r_{\text {peri }}\end{array}$ & \# of Simulations \\
\hline \multicolumn{7}{|c|}{ "Full-Model" Simulations with Black Holes } \\
\hline $\begin{array}{l}\text { Local } \\
\text { Intermediate }-z \\
\text { High }-z \\
\text { Halo Concentrations } \\
\text { Disk Orientation } \\
\text { Orbital Configuration }\end{array}$ & $\begin{array}{c}80,115,160,226,320,500 \\
80,115,160,226,320,500 \\
115,160,226,320,500 \\
160 \\
160 \\
160\end{array}$ & $\begin{array}{c}0 \\
2,3 \\
6 \\
0 \\
0 \\
0\end{array}$ & $\begin{array}{l}0.4,0.8 \\
0.4,0.8 \\
0.4,0.8 \\
0.4 \\
0.4 \\
0.4\end{array}$ & $\begin{array}{l}0.25,1.0 \\
0.25,1.0 \\
0.25,1.0 \\
1.0 \\
1.0 \\
1.0\end{array}$ & $\begin{array}{c}2 r_{\mathrm{d}} \\
2 r_{\mathrm{d}} \\
2 r_{\mathrm{d}} \\
2 r_{\mathrm{d}} \\
\text { Table } 2 \\
\text { Table 2 }\end{array}$ & $\begin{array}{c}24 \\
48 \\
20 \\
5 \\
14 \\
18\end{array}$ \\
\hline \multicolumn{7}{|c|}{ Dissipational Simulations } \\
\hline $\begin{array}{l}\text { Local } \\
\text { Intermediate }-z \\
\text { High }-z \\
f_{\text {gas }} \text { Runs }\end{array}$ & $\begin{array}{c}80,115,160,226,320,500 \\
80,115,160,226,320,500 \\
115,160,226,320,500 \\
80,115,160,226,320,500\end{array}$ & $\begin{array}{c}0 \\
2,3 \\
6 \\
0\end{array}$ & $\begin{array}{c}0.4,0.8 \\
0.4,0.8 \\
0.4,0.8 \\
0.01,0.025,0.05 \\
0.1,0.2,0.4\end{array}$ & $\begin{array}{l}0.25,1.0 \\
0.25,1.0 \\
0.25,1.0 \\
0.25,1.0\end{array}$ & $\begin{array}{l}2 r_{\mathrm{d}} \\
2 r_{\mathrm{d}} \\
2 r_{\mathrm{d}} \\
2 r_{\mathrm{d}}\end{array}$ & $\begin{array}{l}24 \\
48 \\
20 \\
72\end{array}$ \\
\hline \multicolumn{7}{|c|}{ Dissipationless Simulations } \\
\hline $\begin{array}{l}\text { Local } \\
\text { Intermediate }-z \\
\text { High- } z \\
\text { Wide Orbit } \\
\text { Wide Orbit, Int. }-z \\
\text { Wide Orbit, High }-z \\
\text { Bulge, Local } \\
\text { Bulge, Intermediate- } z \\
\text { Bulge, High- }-z \\
\text { High-Res }\end{array}$ & $\begin{array}{l}80,115,160,226,320,500 \\
80,115,160,226,320,500 \\
80,115,160,226,320,500 \\
80,115,160,226,320,500 \\
80,115,160,226,320,500 \\
80,115,160,226,320,500 \\
80,115,160,226,320,500 \\
80,115,160,226,320,500 \\
80,115,160,226,320,500 \\
80,115,160,226,320,500\end{array}$ & $\begin{array}{c}0 \\
2,3 \\
6 \\
0 \\
2,3 \\
6 \\
0 \\
2,3 \\
6 \\
0\end{array}$ & $\begin{array}{l}0.0 \\
0.0 \\
0.0 \\
0.0 \\
0.0 \\
0.0 \\
0.0 \\
0.0 \\
0.0 \\
0.0\end{array}$ & $\begin{array}{l}- \\
- \\
- \\
- \\
- \\
- \\
- \\
- \\
- \\
-\end{array}$ & $\begin{array}{c}2 r_{\mathrm{d}} \\
2 r_{\mathrm{d}} \\
2 r_{\mathrm{d}} \\
0.4 R_{\mathrm{vir}} \\
0.4 R_{\mathrm{vir}} \\
0.4 R_{\mathrm{vir}} \\
2 r_{\mathrm{d}} \\
2 r_{\mathrm{d}} \\
2 r_{\mathrm{d}} \\
2 r_{\mathrm{d}}\end{array}$ & $\begin{array}{c}6 \\
12 \\
6 \\
6 \\
12 \\
6 \\
6 \\
12 \\
6 \\
6\end{array}$ \\
\hline \multicolumn{7}{|c|}{ Spheroid Simulations } \\
\hline $\begin{array}{l}\text { Local } \\
\text { Wide/Elliptical Orbit } \\
\text { Full-Model Re-mergers }\end{array}$ & $\begin{array}{c}80,115,160,226,320,500 \\
80,115,160,226,320,500 \\
80,115,160,226\end{array}$ & $\begin{array}{l}0 \\
0 \\
0 \\
\end{array}$ & $\begin{array}{l}0.0 \\
0.0 \\
0.4 \\
\end{array}$ & $\begin{array}{c}- \\
- \\
0.25\end{array}$ & $\begin{array}{c}0.025 R_{\text {vir }} \\
0.4 R_{\text {vir }} \\
0.05 R_{\text {vir }} \\
\end{array}$ & $\begin{array}{l}6 \\
6 \\
4 \\
\end{array}$ \\
\hline
\end{tabular}

Following Robertson et al. (2005b), we scale the progenitor galaxy properties to approximate the structure of disk galaxies appropriate for redshifts $z=0,2,3$, and 6 . Varying the progenitor galaxies in this manner enables us to determine the impact of redshift-dependent galaxy properties on the scaling laws of ellipticals. Keeping the virial velocity $V_{\text {vir }}$ fixed with redshift, we scale the progenitor virial mass and virial radius using the relations

$$
\begin{gathered}
M_{\mathrm{vir}}=\frac{V_{\mathrm{vir}}^{3}}{10 G H(z)} \\
R_{\mathrm{vir}}=\frac{V_{\mathrm{vir}}}{10 H(z)},
\end{gathered}
$$

where $H(z)$ is the Hubble parameter. To suitably resolve the forces between particles in models of higher-redshift systems, we reduce the gravitational smoothing by $(1+z)^{-1}$. The halo concentrations also vary with redshift and mass according to Equation (3) and the disk scalelengths decrease with redshift through their dependence on $R_{\mathrm{vir}}$ and $C_{\mathrm{vir}}$. We note that the redshift-dependence of disk scalelengths agrees well with the distribution of disk scalelengths seen out to redshift $z \approx 1$ (e.g. Ravindranath et al. 2004; Barden et al. 2005).

At each redshift, we consider three separate types of dissipationless disk mergers. First, we examine equal mass mergers of pure disk galaxies on prograde-prograde coplanar parabolic orbits with the pericentric passage distance set to $r_{\text {peri }}=2 r_{\mathrm{d}}$. The galaxies each contain 60,000 dark matter and 80,000 stellar disk particles. Second, we repeat each merger with a wider parabolic orbit increased to $r_{\text {peri }}=0.4 R_{\mathrm{vir}}$ to judge the effect of increased orbital angular momentum on the dissipationless merger remnants. In addition, we repeat each nearly-radial merger with bulge components included in the galaxies with mass fraction $m_{\mathrm{b}} \equiv M_{\text {bulge }} / M_{\mathrm{vir}}=0.1367$ to match the Milky Way-like model used in Springel et al. (2005). We model the bulges with a Hernquist (1990) density profile form (see Equation 4) where we set the bulge scalelength $b=0.2 r_{\mathrm{d}}$. Each bulge contains 20,000 particles, with the number of disk particles reduced to 60,000 to maintain the same mass resolution. Finally, we re-run all the pure disk simulations at redshift $z=0$ with higher resolution dissipationless models with 180,000 dark matter particles and 120,000 disk particles to examine issues related to numerical resolution. While we discuss these tests in more detail in \$4 we note here that the large set of dissipationless simulations produces results very consistent with the restricted set of higher resolution runs. In all we perform 78 dissipationless simulations, and we provide a complete listing in Table 1

\subsection{Dissipational Disk Simulations}

To gauge the impact of dissipational gas physics on the properties of merger remnants, we perform a suite of disk galaxy mergers that include gas cooling, star formation, and supernova feedback. The dissipational disk progenitors contain exponential gaseous and stellar disks and Hernquist (1990) dark matter halos, with their disk sizes determined by the Mo et al. (1998) formalism as described in $\$ 3.1$ The vertical structure of the gaseous disks are determined by an inte- 
TABLE 2. ORBITAL VARIATIONS

\begin{tabular}{|c|c|c|c|c|c|}
\hline Models & $\begin{array}{c}\theta_{1} \\
{[\mathrm{deg}]}\end{array}$ & $\begin{array}{c}\phi_{1} \\
{[\mathrm{deg}]}\end{array}$ & $\begin{array}{c}\theta_{2} \\
{[\mathrm{deg}]}\end{array}$ & $\begin{array}{c}\phi_{2} \\
{[\mathrm{deg}]}\end{array}$ & $\begin{array}{c}r_{\text {peri }} \\
{\left[h^{-1} \mathrm{kpc}\right]}\end{array}$ \\
\hline \multicolumn{6}{|c|}{ Disk Orientations } \\
\hline$b$ & 180 & 0 & 0 & 0 & 5.0 \\
\hline$c$ & 180 & 0 & 180 & 0 & 5.0 \\
\hline$d$ & 90 & 0 & 0 & 0 & 5.0 \\
\hline$e$ & 30 & 60 & -30 & 45 & 5.0 \\
\hline$f$ & 60 & 60 & 150 & 0 & 5.0 \\
\hline$g$ & 150 & 0 & -30 & 45 & 5.0 \\
\hline h & 0 & 0 & 0 & 0 & 5.0 \\
\hline$i$ & 0 & 0 & 71 & 30 & 5.0 \\
\hline$j$ & -109 & 90 & 71 & 90 & 5.0 \\
\hline$k$ & -109 & -30 & 71 & -30 & 5.0 \\
\hline$l$ & -109 & 30 & 180 & 0 & 5.0 \\
\hline$m$ & 0 & 0 & 71 & 90 & 5.0 \\
\hline$n$ & -109 & -30 & 71 & 30 & 5.0 \\
\hline$o$ & -109 & 30 & 71 & -30 & 5.0 \\
\hline$p$ & -109 & 90 & 180 & 0 & 5.0 \\
\hline \multicolumn{6}{|c|}{ Orbital Configurations } \\
\hline$e 1$ & 30 & 60 & -30 & 45 & 2.5 \\
\hline$e 2$ & 30 & 60 & -30 & 45 & 10.0 \\
\hline$e 3$ & 30 & 60 & -30 & 45 & 15.0 \\
\hline$e 4$ & 30 & 60 & -30 & 45 & 20.0 \\
\hline$e 5$ & 30 & 60 & -30 & 45 & 40.0 \\
\hline$e 6$ & 30 & 60 & -30 & 45 & 30.0 \\
\hline$h 1$ & 0 & 0 & 0 & 0 & 2.5 \\
\hline$h 2$ & 0 & 0 & 0 & 0 & 10.0 \\
\hline$h 3$ & 0 & 0 & 0 & 0 & 15.0 \\
\hline$h 4$ & 0 & 0 & 0 & 0 & 20.0 \\
\hline$h 5$ & 0 & 0 & 0 & 0 & 40.0 \\
\hline$h 6$ & 0 & 0 & 0 & 0 & 30.0 \\
\hline$k 1$ & -109 & -30 & 71 & -30 & 2.5 \\
\hline$k 2$ & -109 & -30 & 71 & -30 & 10.0 \\
\hline$k 3$ & -109 & -30 & 71 & -30 & 15.0 \\
\hline$k 4$ & -109 & -30 & 71 & -30 & 20.0 \\
\hline$k 5$ & -109 & -30 & 71 & -30 & 40.0 \\
\hline$k 6$ & -109 & -30 & 71 & -30 & 30.0 \\
\hline
\end{tabular}

gral constraint from the surface mass density and the requirement of hydrostatic equilibrium within the galaxy potential.

The thermal properties of the gas are determined using the multiphase interstellar medium (ISM) model of Springel \& Hernquist (2003). Star formation is prescribed in the manner of Springel \& Hernquist (2003), constrained to approximate the Schmidt (1959) law for disk galaxies as measured by Kennicutt (1998), including a density threshold. Below this threshold the gas is modeled as a single-phase medium which is not star-forming. Dense gas above the threshold is modeled as a hybrid of cold, dense clouds embedded in a hot, diffuse medium as envisioned by McKee \& Ostriker (1977). The temperature of the hot phase is set by supernova feedback and the efficiency of cloud evaporation, and has an energy per unit mass that far exceeds the cold phase. Even though most of the gas by mass is cold, the high temperature of the hot phase more than compensates for its small mass fraction, acting to pressurize the star-forming gas, and leading to an effective equation of state $P_{\text {eff }}(\rho)$ that is stiffer than isothermal (for a numerical fit, see Robertson et al. 2004). The multiphase model of Springel \& Hernquist (2003) has been generalized by Springel et al. (2005) to allow for an effective equation of state parameter $q_{\mathrm{EOS}}$ that linearly interpolates between an isothermal gas $\left(q_{\mathrm{EOS}}=0\right)$ and the fully-pressurized multiphase ISM model $\left(q_{\mathrm{EOS}}=1\right)$. Increasing $q_{\text {EOS }}$ improves the dynamical stability of the gas and can prevent Toomre (1964) instability even in gas-rich systems (Springel \& Hernquist 2003; Robertson et al. 2004; Springel \& Hernquist 2005; Robertson et al. 2005a).

For our dissipational models, we re-run the $V_{\text {vir }}=80-500$ $\mathrm{km} \mathrm{s}^{-1}$ pure disk merger simulations from $\$ 3.1$ with two gas fractions of $f_{\text {gas }}=0.4,0.8$, each with two equation of state parameters $q_{\mathrm{EOS}}=0.25,1.0$, at redshifts $z=0,2,3$, and 6 . Each progenitor galaxy has 60,000 dark matter particles, 40,000 stellar disk particles, and 40,000 gas particles. The systems are merged on prograde-prograde parabolic coplanar orbits with $r_{\text {peri }}=2 r_{\mathrm{d}}$. For an equation of state parameter $q_{\mathrm{EOS}}=0.25$ we also systematically vary the gas fraction of $z=0$ progenitors using $f_{\text {gas }}=0.01,0.025,0.05,0.1,0.2$ and 0.4 . Our dissipational simulation category has a total of 164 runs, with the complete list of simulations provided in Table 1

\subsection{Full-Model Disk Simulations}

Our full-model category simulations include the complete physical model presented in Springel et al. (2005), accounting for gas cooling, star formation, supernova feedback, the Springel \& Hernquist (2003) ISM model described in $\$ 3.2$ and a prescription for supermassive black hole growth and feedback. The supermassive black holes are included as "sink" particles, with seed masses of $10^{5} h^{-1} M_{\odot}$. The black holes are allowed to grow according to spherical Bondi-Hoyle-Lyttleton accretion (Hovle \& Lyttleton 1939; Bondi \& Hoyle 1944; Bondi 1952). The mass accretion rate $\dot{M}$ is determined from the density and sound speed of the gas near the black hole. A fraction $\epsilon_{\mathrm{f}}=0.1$ of the accretion rate is radiatively released, of which a fraction $\eta_{\text {therm }}=0.05$ is coupled as thermal feedback into gas within an SPH kernel smoothing length of the black hole. The strength of the thermal coupling is comparable to the thermal feedback coupling of supernova energy used in cosmological simulations (e.g. Abadi et al. 2003), and reproduces the $M_{\mathrm{BH}}-\sigma$ relation observed locally (Di Matteo et al. 2005). Using the same fullmodel simulations presented here, Robertson et al. (2005b) have also demonstrated that this model for black hole accretion and feedback should preserve the power-law scaling of the $M_{\mathrm{BH}}-\sigma$ relation between redshifts $z=0-6$.

The full-model simulations augment the pure disk dissipational merger simulations from $\$ 3.2$ with supermassive black hole growth as described above. Each progenitor galaxy has 40,000 stellar disk particles and 40,000 gas particles, and are merged on prograde-prograde parabolic coplanar orbits with $r_{\text {peri }}=2 r_{\mathrm{d}}$. The models are calculated for $V_{\mathrm{vir}}=80-500 \mathrm{~km}$ $\mathrm{s}^{-1}$ galaxies at $z=0,2$, and 3 and $V_{\mathrm{vir}}=115-500 \mathrm{~km} \mathrm{~s}^{-1}$ galaxies at $z=6$. Simulations are performed with gas fractions of $f_{\text {gas }}=0.4,0.8$ each with equation of state parameters of $q_{\mathrm{EOS}}=0.25,1.0$. Furthermore, we run 14 variations of the $V_{\mathrm{vir}}=160 \mathrm{~km} \mathrm{~s}^{-1}$ progenitor merger where we change the disk orientation according to the method of Barnes (1992) to characterize the effects of disk alignment (see Table 2), and run an additional 18 simulations where for 3 different orientations we vary the pericentric passage distance. We also run a set of 5 additional simulations of the $V_{\text {vir }}=160 \mathrm{~km} \mathrm{~s}^{-1}$ halo where we simulate dark matter halo concentrations of $C_{\mathrm{vir}}=5,7,9$, 11 , and 13. In all, we perform a total of 129 full-model simulations and a complete list of these runs is provided in Table 1

\subsection{Spheroid Simulations}

To explore the properties of remnants formed by the merging of spheroidal systems, we also perform a suite of equal 
mass spheroid-spheroid mergers. The spheroid progenitors consist of stellar spheroids embedded in dark matter halos, both having Hernquist (1990) profiles. We assume a stellar mass fraction of $f_{\star}=0.05$ and the concentrations of the halos are adjusted to account for the mass-dependence measured in cosmological simulations (see Section 3.1). The sizes of the stellar spheroids are set to follow the Shen et al. (2003) $R_{\mathrm{e}}-M_{\star}$ relation for massive galaxies as

$$
R_{\mathrm{e}}=4.16\left(\frac{M_{\star}}{10^{11} M_{\odot}}\right)^{0.56} \mathrm{kpc},
$$

(see also Boylan-Kolchin et al. 2005). We vary the circular velocity of the halos between $V_{\text {vir }}=80-500 \mathrm{~km} \mathrm{~s}^{-1}$. The spheroid galaxies merge on either nearly-radial parabolic orbits with $r_{\text {peri }}=0.025 R_{\text {vir }}$ or wide elliptical orbits with ellipticity $\epsilon=0.5$ and $r_{\text {peri }}=0.4 R_{\text {vir }}$. Each spheroid progenitor has 1,200,000 dark matter and 80,000 stellar particles. A complete list of the spheroid-spheroid mergers is provided in Table 1.

To determine the impact subsequent re-merging between ellipticals formed from disk galaxy mergers might have on the structural properties of the remnants, we also re-merge disk galaxy remnants from the $z=0$ full-model simulations. These remnants are merged on parabolic orbits with $r_{\text {peri }}=0.05 R_{\mathrm{vir}}$, where $R_{\text {vir }}$ is the virial radius of the original disk progenitor. We re-merge remnants with progenitor galaxy circular velocities in the range $V_{\text {vir }}=80-226 \mathrm{~km} \mathrm{~s}^{-1}$. A complete list of the re-merger simulations is provided in Table 1

\subsection{Analysis}

Each simulation is evolved until the merger is complete and the remnants are fully relaxed, requiring integrations of typically 2-4 Gyr. The remnants are then kinematically analyzed by measuring the half-mass stellar effective radii $R_{\mathrm{e}}$, the average one- dimensional velocity dispersion $\sigma$ within a circular aperture of radius $R_{\mathrm{e}}$, and the average stellar surface mass density $I_{\mathrm{e}}$ measured within $R_{\mathrm{e}}$ as $I_{\mathrm{e}} \equiv M_{\star}\left(r<R_{\mathrm{e}}\right) / \pi R_{\mathrm{e}}^{2}$. The quantities $R_{\mathrm{e}}, \sigma$, and $I_{\mathrm{e}}$ are averaged over 100 random sight lines to the remnant.

Once the FP parameters $R_{\mathrm{e}}, \sigma$, and $I_{\mathrm{e}}$ are determined, we employ the direct fitting method of Bernardi et al. (2003a) to determine the best-fit FP scalings. The direct fitting method seeks to minimize

$$
\Delta=\log R_{\mathrm{e}}-\alpha \log \sigma-\beta \log I_{\mathrm{e}}-\delta,
$$

where $\alpha$ and $\beta$ are the FP scaling indices defined by Equation (11). The minimization of $\Delta$ requires

$$
\begin{gathered}
\alpha=\frac{\left(\sigma_{\mathrm{II}}^{2} \sigma_{\mathrm{RV}}^{2}-\sigma_{\mathrm{IR}}^{2} \sigma_{\mathrm{IV}}^{2}\right)}{\left(\sigma_{\mathrm{II}}^{2} \sigma_{\mathrm{VV}}^{2}-\sigma_{\mathrm{II}}^{4}\right)} \\
\beta=\frac{\left(\sigma_{\mathrm{VV}}^{2} \sigma_{\mathrm{IR}}^{2}-\sigma_{\mathrm{RV}}^{2} \sigma_{\mathrm{IV}}^{2}\right)}{\left(\sigma_{\mathrm{II}}^{2} \sigma_{\mathrm{VV}}^{2}-\sigma_{\mathrm{IV}}^{4}\right)} \\
\delta=\left\langle\log R_{\mathrm{e}}\right\rangle-\alpha\langle\log \sigma\rangle-\beta\left\langle\log I_{\mathrm{e}}\right\rangle,
\end{gathered}
$$

where the average $\langle\log X\rangle$ over the $N$ data samples considered is defined as

$$
\langle\log X\rangle \equiv \sum_{i} \log X_{i} / N
$$

\begin{tabular}{|c|c|c|c|c|c|}
\hline \multirow[t]{2}{*}{ Models } & \multicolumn{4}{|c|}{ Fundamental Plane } & \multirow{2}{*}{$\begin{array}{c}R_{\mathrm{e}}-M_{\star} \\
\mu\end{array}$} \\
\hline & $\alpha$ & $\beta$ & $\lambda$ & $\left\langle\Delta^{2}\right\rangle^{1 / 2}$ & \\
\hline Dissipationless & 2.00 & 1.01 & $1.00 \pm 0.01$ & 0.018 & $0.45 \pm 0.03$ \\
\hline Dissipational & 1.58 & 0.80 & $0.80 \pm 0.01$ & 0.065 & $0.57 \pm 0.02$ \\
\hline Full-Model & 1.55 & 0.82 & $0.79 \pm 0.01$ & 0.062 & $0.57 \pm 0.02$ \\
\hline \multicolumn{6}{|c|}{ Gas Fraction $f_{\text {gas }}$ Runs } \\
\hline$f_{\text {gas }}=0.01$ & 1.81 & 0.75 & $0.97 \pm 0.01$ & 0.009 & $0.41 \pm 0.01$ \\
\hline$f_{\text {gas }}=0.025$ & 2.11 & 0.74 & $0.96 \pm 0.01$ & 0.011 & $0.41 \pm 0.01$ \\
\hline$f_{\text {gas }}=0.05$ & 2.07 & 0.64 & $0.95 \pm 0.01$ & 0.011 & $0.41 \pm 0.01$ \\
\hline$f_{\text {gas }}=0.1$ & 2.01 & 0.61 & $0.92 \pm 0.01$ & 0.014 & $0.42 \pm 0.01$ \\
\hline$f_{\text {gas }}=0.2$ & 1.89 & 1.20 & $0.89 \pm 0.02$ & 0.024 & $0.44 \pm 0.01$ \\
\hline$f_{\mathrm{gas}}=0.4$ & 1.64 & 1.07 & $0.83 \pm 0.02$ & 0.033 & $0.51 \pm 0.03$ \\
\hline
\end{tabular}

and the co-variant dispersion is defined as

$$
\sigma_{\mathrm{XY}}^{2}=\sum_{i}\left(\log X_{i}-\langle\log X\rangle\right)\left(\log Y_{i}-\langle\log Y\rangle\right) / N .
$$

TABLE 3. BEST-Fit SCALINGS

The mean-squared scatter about the direct best-fit plane can then be characterized by the quantity

$$
\begin{array}{r}
\left\langle\Delta^{2}\right\rangle=\left(\sigma_{\mathrm{II}}^{2} \sigma_{\mathrm{RR}}^{2} \sigma_{\mathrm{VV}}^{2}-\sigma_{\mathrm{II}}^{2} \sigma_{\mathrm{RV}}^{4}-\sigma_{\mathrm{RR}}^{2} \sigma_{\mathrm{IV}}^{4}\right. \\
\left.-\sigma_{\mathrm{VV}}^{2} \sigma_{\mathrm{IR}}^{4}+2 \sigma_{\mathrm{IR}}^{2} \sigma_{\mathrm{IV}}^{2} \sigma_{\mathrm{RV}}^{2}\right) \\
\times\left(\sigma_{\mathrm{II}}^{2} \sigma_{\mathrm{VV}}^{2}-\sigma_{\mathrm{IV}}^{4}\right)^{-1}
\end{array}
$$

When appropriate we compare the quantity $\left\langle\Delta^{2}\right\rangle^{1 / 2}$ with the scatter determined from observational samples.

The remnant properties are also compared to the $R_{\mathrm{e}}-M_{\star}$ relation, which has been measured observationally in the SDSS (Shen et al. 2003) and may be represented by the power-law form

$$
R_{\mathrm{e}} \propto M_{\star}^{\mu} .
$$

The $R_{\mathrm{e}}-M_{\star}$ relation allows for a useful comparison of remnant sizes for simulations with differing angular momenta, progenitor redshifts, ISM physics, or gas dissipation. In addition, we find that the relative location of remnants in the FP can often be related to the impact of different physical processes on the effective radii. The $R_{\mathrm{e}}-M_{\star}$ relation also serves as a useful calibration for our method to measure the FP properties of the remnants. As mentioned in $\$ 3.4$ a subset of our simulation suite involves the merging of equilibrium models of spheroids initialized to satisfy the Shen et al. (2003) relation. As discussed in further detail in $\$ 4.4$ the analysis technique used to measure the FP properties of remnants accurately recovers the Shen et al. (2003) relation when applied to the spheroid model progenitors and affirms our ability to determine simulated remnant properties with reasonable fidelity.

Typically the root-mean-squared variation in the velocity dispersion and effective radius along different lines-of-sight to a given remnant are $\Delta \sigma \approx 20 \mathrm{~km} \mathrm{~s}^{-1}$ and $\Delta R_{\mathrm{e}} \approx 0.5 h^{-1} \mathrm{kpc}$, respectively, for remnants formed through mergers of $V_{\mathrm{vir}}=$ $160 \mathrm{~km} \mathrm{~s}^{-1}$ progenitors. The magnitude of the line-of-sight variation scales with the velocity dispersion or effective radius, but the fractional line-of-sight variation remains similar with $\Delta \sigma \approx 0.1\langle\sigma\rangle$ and $\Delta R_{\mathrm{e}} \approx 0.1\left\langle R_{\mathrm{e}}\right\rangle$ roughly independent of progenitor mass. The combined quantity $\sigma^{2} I_{\mathrm{e}}^{-1}$ has a typical fractional line-of-sight variation of $\Delta \sigma^{2} I_{\mathrm{e}}^{-1} \approx 0.3\left\langle\sigma^{2} I_{\mathrm{e}}^{-1}\right\rangle$ roughly independent of progenitor mass and under this variation galaxies tend to move along relatively short trajectories in the $R_{\mathrm{e}} \sigma I_{\mathrm{e}}$-space that do not deviate far from the mean FP. Certain orbits, such as the head-on orbit considered for spheroidal mergers in 4.4 can induce larger line-of-sight variations but the average variations reported above are much more frequent 
in our remnants. The impact of these relatively small errors on estimates of the power-law correlations of the FP and $R_{\mathrm{e}}-M_{\star}$ relations are minor and comparable to deviations induced by either orbital variations or the structural properties of progenitors, and therefore additional scatter induced by the line-ofsight variations of individual systems is not considered by our subsequent analysis. However, when plotting either the FP or the $R_{\mathrm{e}}-M_{\star}$ relations, we provide sample error bars to show the rough deviations any single galaxy could make from their mean positions due to line-of-sight variations in $\sigma$ or $R_{\mathrm{e}}$.

\subsection{Comparison with Observations}

We adopt the approach of plotting the FP properties of simulated remnants in the $R_{\mathrm{e}}-\sigma^{2} I_{\mathrm{e}}^{-1}$ virial plane coordinate system. The tilt of the FP relative to the virial plane can be quantified through the power-law relation

$$
R_{\mathrm{e}} \propto\left(\sigma^{2} I_{\mathrm{e}}^{-1}\right)^{\lambda}
$$

where $\lambda=1$ indicates an alignment of the FP with the virial plane. When appropriate, we measure this estimate of the FP tilt relative to the virial plane by linear least-squares fitting. Our choice of coordinate systems is not unique, and alternative representations of the FP include the $\kappa$-space coordinate system (Bender et al. 1992) or the best-fit FP coordinates determined by observations in various passbands. The primary advantage of choosing the virial plane coordinate system is the easily determined tilt, which provides a gauge of possible variations in the central total-to-stellar mass $M_{\text {total }} / M_{\star}$ or kinematic nonhomology of remnants as a function of galaxy mass.

For our purposes, we choose not to use stellar population synthesis to compare with determinations of the FP in optical passbands. Observations indicate that trends in the $M / L$ ratio owing to stellar population effects (e.g. age or metallicity) as a function of galaxy mass or luminosity will contribute significantly to the FP tilt, especially at short-wavelengths (for a recent result on this issue, see Cappellari et al. 2005). Observations have also determined that elliptical galaxies are typically old and their stellar populations redden passively with time (e.g. Bender et al. 1996; van Dokkum \& Franx 1996). These constraints imply that to properly recover the shortwavelength photometric FP, precise information on the stellar age, metallicity, and formation-redshift distribution of elliptical galaxies as a function of stellar mass at $z=0$ must be obtained. We note that knowing only either the formationredshift (e.g. the redshift of the last major gas-rich merger) or mean stellar age of elliptical galaxies may not be sufficient to determine the photometric FP of the entire elliptical galaxy population. Recent surveys indicate that ellipticals typically undergo a major dissipationless merger at redshifts $z<1$ (Bell et al. 2005; van Dokkum 2005), and, in principle, such mergers may induce galaxy mass-dependent tilt from structural effects that are disjoint from $M / L$ effects from stellar populations that characteristically predate those events. Clearly, applying stellar population synthesis models to elliptical galaxies produced in individual galaxy merger simulations to produce simulated photometric FP scalings without attempting to correct for the cosmologically-determined properties of the real elliptical population is likely too naive. Furthermore, comparing directly stellar-mass FP scalings determined from simulations with short-wavelength (e.g. $B$ band or Sloan $g$-band) photometric FP scalings should be performed with extreme caution as the short-wavelength FP may have additional sources of tilt not present in the stellar-mass FP.

With these concerns in mind, our stellar-mass FP results will be compared with the near-infrared (IR) FP determined by Pahre et al. (1998b). While, as Pahre et al. (1998b) note, tilt owing to stellar population effects may still be present and is not tightly constrained, structural or dynamical nonhomologies can contribute significantly to the FP scaling in the near-IR. The $K$-band magnitudes of stellar populations of a given age are less influenced by metallicity than their optical passband luminosities (e.g. Bruzual \& Charlot 2003), and typical $K$-band mass-to-light ratios are within $\approx 35 \%$ of unity for stellar populations formed at redshifts $0.75<z<5.0$. These properties make comparisons between the stellar-mass and near-IR FP scalings somewhat more sensible than comparisons with shorter-wavelength photometric FP scalings, though not ideal.

In principle, a more straight-forward comparison would be to use high-resolution cosmological simulations of galaxy formation to probe the fundamental plane with simultaneously accounting for metallicity and stellar age effects. Previous attempts to compare results of cosmological simulations for the scaling laws of elliptical galaxies with observations have been made (e.g. Sáiz et al. 2004), but with spatial resolution some $\approx 20$ times worse than the isolated merger simulations presented here. Such spatial resolution is larger than the effective radii of even moderately-sized $\left(M_{\mathrm{r}} \geq 21\right)$ early-type galaxies. However, the metallicities and ages of the stellar populations of galaxies produced in cosmological simulations can be estimated throughout their formation, and the results of stellar population synthesis modeling would therefore be easy to interpret.

We mention here that semi-analytic techniques could be combined with the results of high-resolution merger simulations in an attempt to account for the redshiftdependent formation of the elliptical galaxy population. For example, Robertson et al. (2005a) used the results of Hopkins et al. (2005a), who inferred a redshift-dependent black hole mass function from the quasar luminosity function, to determine the influence of the redshift-dependent formation times of elliptical galaxies on the $M_{\mathrm{BH}}-\sigma$ relation. Using the results from Robertson et al. (2005a) and Hopkins et al. (2005a), Hopkins et al. (2005b) combined the redshift-dependent properties of galaxy remnants, the $M_{\mathrm{BH}}-\sigma$ relation, and the redshift-dependent black hole mass function to model the evolution of the red-galaxy luminosity function and color-magnitude relation. A combination of the results from Hopkins et al. (2005b) and the stellar-mass FP relation presented here could be used to account for the effects of color-magnitude evolution on the photometric FP, but would likely involve other assumptions beyond those employed here and we defer such analysis for future work.

\section{RESULTS}

Below, we present the FP and $R_{\mathrm{e}}-M_{\star}$ relations for the dissipationless, dissipational, full-model, and spheroidal merger simulations. For each FP and $R_{\mathrm{e}}-M_{\star}$ relation, we list the best fit scalings in Table 3 with 1- $\sigma$ errors on their power-law slopes (i.e. $\lambda$ for the FP and $\mu$ for the $R_{\mathrm{e}}-M_{\star}$ ).

\subsection{Dissipationless Disk Simulations}

The merging of the dissipationless disks described in $\$ 3.1$ produces a FP relation similar to the virial scaling. Figure 11 shows the location of dissipationless merger remnants in 


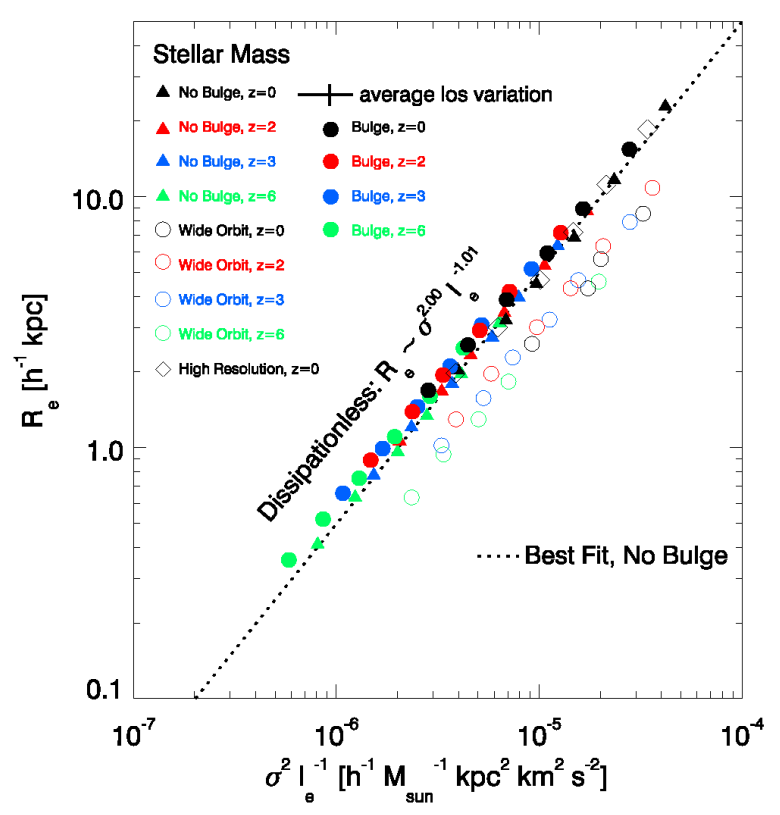

FIG. 1.- Fundamental Plane (FP) relation produced by the merging of dissipationless disk galaxy models appropriate for redshifts $z=0$ (black), $z=2$ (red), $z=3$ (blue), and $z=6$ (green) on nearly radial, parabolic orbits. All models include dark matter halos. The dissipationless merging of pure disk models (solid triangles) and disk models with bulges (solid circles) produce similar FP relations nearly parallel to the plane defined by the virial relation. Increasing the angular momentum of the orbit by lengthening the pericentric passage distance of the orbit produces an offset in the FP by increasing the effective radius of the remnants (open circles), but the systems still obtain a FP scaling similar to the virial plane. Select higher resolution runs closely follow the FP delineated by their lower resolution counter-parts (open diamonds). For comparison, the best leastsquares fit to the FP of pure disk merger remnants is plotted (dotted line). Also shown is the mean deviation induced by line-of-sight variations in projected quantities for a given remnant (detached error bars).

the virial coordinate system, and plots remnants from progenitors appropriate for various redshifts. The best-fit FP scalings produced by pure disk systems (solid triangles) are $\alpha=2.00, \beta=1.01$, with almost no tilt relative to the virial plane $(\lambda=1.00)$. Individually, progenitors at each of the four simulated redshifts produce merger remnants also closely aligned with the virial plane $(\lambda=0.97-1.03)$. Including stellar bulges in the progenitors (solid circles) produces a similar FP scaling ( $\alpha=1.95, \beta=0.98 ; \lambda=0.97)$. A more substantial change in the FP is realized by increasing the pericentric passage distance of the encounters from $2 r_{\mathrm{d}}$ to $0.4 R_{\mathrm{vir}}$ (open circles), which correspondingly increases the total angular momenta of the merging systems. Remnants produced in the dissipationless wide orbit mergers typically have larger effective radii, inducing an offset of $\Delta \log R_{\mathrm{e}} \sim-0.4$ in the FP. However, the wide orbit FP scalings $(\alpha=1.97, \beta=1.04 ; \lambda=0.97)$ are still very similar to both the nearly radial orbit FP and virial scalings. Increasing the number of dark matter particles per halo to 180,000 in the progenitors (open diamonds) has very little effect on the resulting FP, suggesting our results are not strongly influenced by our numerical resolution.

The $R_{\mathrm{e}}-M_{\star}$ relation produced by merging dissipationless

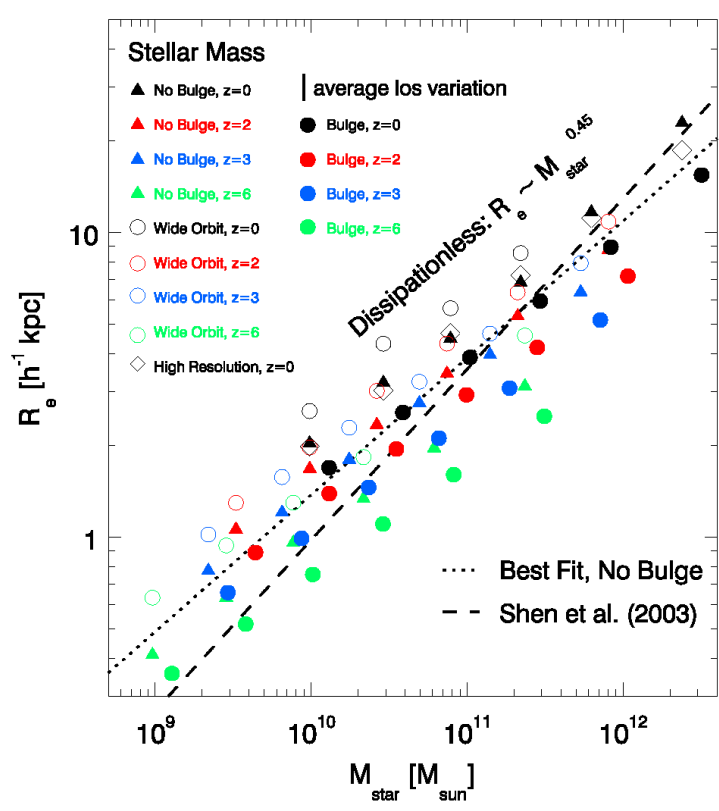

FIG. 2.- Effective radius $R_{\mathrm{e}}-$ stellar mass $M_{\star}$ relation produced by the merging of dissipationless disk galaxy models appropriate for redshifts $z=0$ (black), $z=2$ (red), $z=3$ (blue), and $z=6$ (green) on nearly radial, parabolic orbits. All models include dark matter halos. The dissipationless merging of pure disk models (solid triangles) and disk models with bulges (solid circles) produce $R_{\mathrm{e}}-M_{\star}$ relations shallower than that measured for massive galaxies in the Sloan Digital Sky Survey (Shen et al. 2003). For comparison, the best least-squares fit to the $R_{\mathrm{e}}-M_{\star}$ relation of pure disk merger remnants is plotted (dotted line). Also shown is the mean deviation induced by line-of-sight variations in projected quantities for a given remnant (detached error bars).

disk progenitors, shown in Figure 2 reflects the trends apparent in the FP those mergers generate and additional features owing to redshift-dependent progenitor properties. The merging of pure disk galaxies (solid triangles) appropriate for various redshifts generates remnants with a shallower mean $R_{\mathrm{e}}-M_{\star}$ relation ( $\mu \approx 0.45$, solid line) than that measured in late-type galaxies in the Sloan Digital Sky Survey (Shen et al. 2003, $\mu \approx 0.56$ ). The remnants also systematically decrease in effective radius with redshift, reflecting the decrease in progenitor disk scalelength and pericentric passage distance. Wider orbit mergers (open circles) with larger angular momenta produce remnants with larger effective radii while galaxies containing bulges (solid circles), and therefore less specific angular momenta, produce remnants with smaller effective radii. These results are consistent with expectations for dissipationless systems where the energy and angular momenta of the model system are manifestly conserved. As a final note for the dissipationless runs, increasing the numerical resolution of the dark matter by $3 \times$ (open diamonds) gives consistent results, suggesting that artificial heating of the stellar component by discreteness effects in the dark matter halo is not strongly influencing the structure of the remnants.

\subsection{Dissipational Disk Simulations}

The merging of gas-rich disk galaxy progenitors including dissipation results in FP and $R_{\mathrm{e}}-M_{\star}$ relations that differ substantially from the analogous relations produced by the merg- 


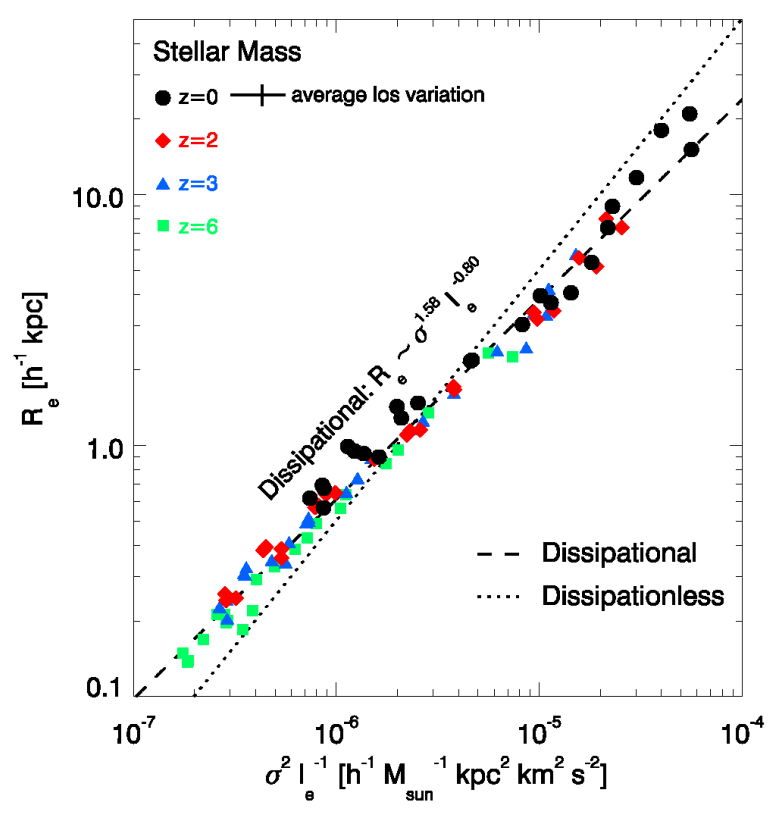

FIG. 3.- Fundamental Plane (FP) relation produced by the merging of gas-rich disk galaxies with dark matter halos, star formation and supernova feedback. Shown are remnants produced by mergers appropriate for redshifts $z=0$ (black circles), $z=2$ (red diamonds), $z=3$ (blue triangles), and $z=6$ (green squares) with nearly radial, parabolic orbits. The dissipational merging of pure disk models produces a FP nearly parallel to the observed infrared FP (Pahre et al. 1998b) and is almost independent of the redshift scalings of the progenitor systems. For comparison, the best least-squares fit to the FP delineated by the remnants is plotted (solid line). Also shown is the mean deviation induced by line-of-sight variations in projected quantities for a given remnant (detached error bars).

ing of dissipationless disks. Figure 3 shows the FP relation generated by the dissipational model disk galaxies appropriately scaled for various redshifts. The dissipational model FP displays a tilt relative to the virial plane $(\lambda=0.8)$, with a scaling $(\alpha=1.58, \beta=0.80)$ similar to the near-IR photometric FP (Pahre et al. 1998b, $\alpha=1.53, \beta=0.79$ ). The dissipational model FP includes remnants produced from progenitors that vary by a factor $2 \times$ in gas fraction $\left(f_{\text {gas }}=0.4,0.8\right)$, including either strongly $\left(q_{\mathrm{EOS}}=1.0\right)$ or weakly $\left(q_{\mathrm{EOS}}=0.25\right)$ pressurized ISM equations-of-state. For gas-rich systems $\left(f_{\text {gas }} \geq 0.4\right)$, the gas fraction and ISM physics of the progenitors have only a slight effect on the FP. The redshift-dependent properties of progenitor systems have little effect on the simulated FP plane, even as the structural properties of the disk galaxies vary substantially with redshift. Combined, varying the gas fraction above $f_{\text {gas }}>0.4$, changing the ISM pressurization dramatically, and scaling the progenitor systems for redshifts $z=0-6$ produce only a small amount of scatter in the dissipational model FP $\left(\left\langle\Delta^{2}\right\rangle^{1 / 2}=0.065\right)$.

The $R_{\mathrm{e}}-M_{\star}$ relation of the remnants, shown in Figure 4 is more strongly influenced by the range of progenitor properties. The smaller progenitor galaxies appropriate for higher redshifts produce smaller remnants, while the less-pressurized ISM models also decrease the effective radii of the remnants. The dissipational model $R_{\mathrm{e}}-M_{\star}$ relation is significantly steeper (solid line, $\mu=0.57$ ) than the relation produced by

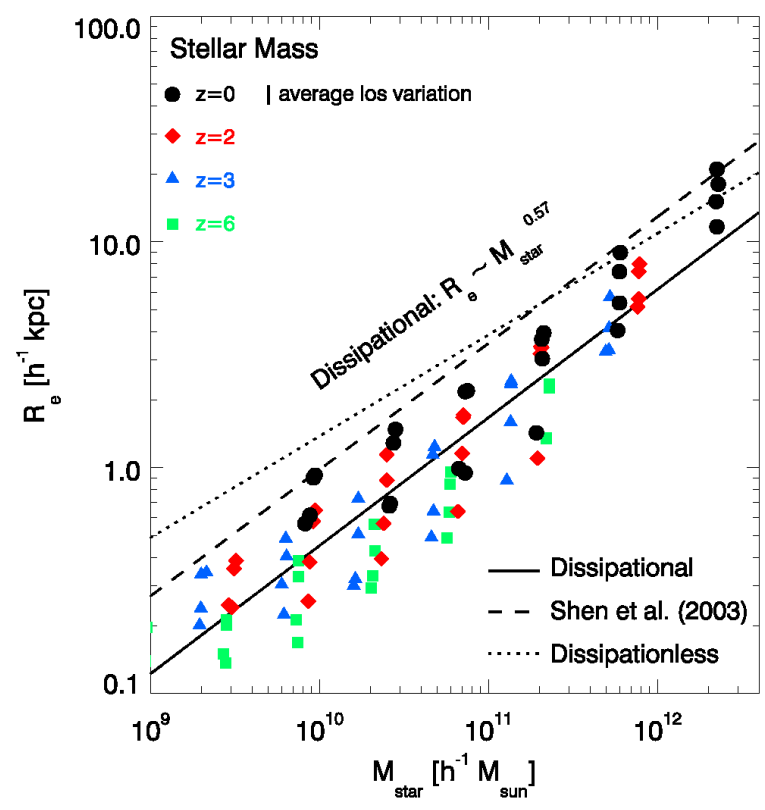

FIG. 4.- Effective radius $R_{\mathrm{e}}-$ stellar mass $M_{\star}$ relation produced by the merging of gas-rich disk galaxies with dark matter halos, star formation and supernova feedback. Shown are remnants produced by mergers appropriate for redshifts $z=0$ (black circles), $z=2$ (red diamonds), $z=3$ (blue triangles), and $z=6$ (green squares) with nearly radial, parabolic orbits. The dissipational merging of pure disk models produces a $R_{\mathrm{e}}-M_{\star}$ relation roughly parallel to that measured for massive galaxies in the Sloan Digital Sky Survey (Shen et al. 2003). For comparison, the best least-squares fit to the $R_{\mathrm{e}}-M_{\star}$ relations delineated by the dissipational simulations (solid line) and dissipationless simulations (dotted line) are plotted. Also shown is the mean deviation induced by line-of-sight variations in projected quantities for a given remnant (detached error bars).

the dissipationless merging of disk galaxies (dotted line, $\mu=$ 0.45 ), and compares well with the relation measured for latetype galaxies in the SDSS (Shen et al. 2003). The dissipational simulations produce an $R_{\mathrm{e}}-M_{\star}$ relation that has a lower $R_{\mathrm{e}}$ normalization than the Shen et al. (2003) relation, but subsequent re-merging and a cosmologically-representative distribution of orbits will likely decrease the discrepancy by increasing $R_{\mathrm{e}}$ at a given stellar mass. Since these the dissipationless remnants lie above the Shen et al. (2003) relation, re-merging and higher angular momentum orbits will only further increase this discrepancy with real elliptical galaxies. While a proper accounting for the distribution of formation redshifts for elliptical galaxies would alter the $R_{\mathrm{e}}-M_{\star}$ relation by weighting the remnants unequally, the mergers of disk galaxies where dissipation is important will nevertheless produce a $R_{\mathrm{e}}-M_{\star}$ relation that is steeper than that produced by dissipationless merging.

\subsection{Full-Model Disk Simulations}

Introducing the effects of black hole feedback through the full-model simulations produces a set of fundamental scaling relations similar to those in dissipational mergers without black holes. The fundamental plane produced by the full-model simulations yields nearly the same FP scalings $(\alpha=1.55, \beta=0.82)$ and tilt $(\lambda=0.79)$ as the dissipational simulations (see $\$ 4.2$, and is similar to the observed near-IR scal- 


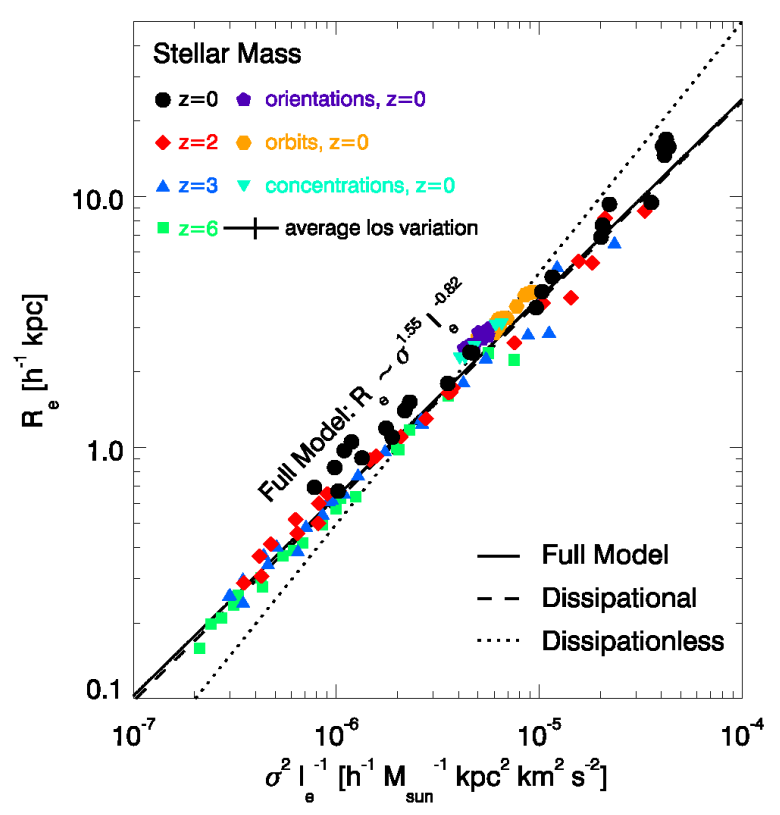

FIG. 5.- Fundamental Plane (FP) relation produced by the merging of gas-rich disk galaxies with dark matter halos, star formation, supernova feedback, and a prescription for feedback from accreting supermassive black holes. Shown are remnants produced by mergers appropriate for redshifts $z=0$ (black circles), $z=2$ (red diamonds), $z=3$ (blue triangles), and $z=6$ (green squares) with nearly radial, parabolic orbits. The merging of pure disk galaxies using our full physical model produces a FP nearly parallel to the observed infrared FP (Pahre et al. 1998b) and nearly coincident with the FP produced by similar simulations without black holes. The FP relation is roughly independent of the redshift scalings of the progenitor systems and the location of remnants within FP is fairly insensitive to a large variety of disk orientations (purple pentagons) and orbital configurations (orange hexagons), as changes in the effective radius are compensated by changes in the velocity dispersion and surface mass density. For comparison, the best least-squares fit to the FP delineated by the remnants is plotted (solid line). Also shown is the mean deviation induced by line-of-sight variations in projected quantities for a given remnant (detached error bars).

ings (Pahre et al. 1998b). These simulated remnants exhibit a scatter about their mean FP of $\left\langle\Delta^{2}\right\rangle^{1 / 2}=0.062$, comparable to both the observed scatter in the FP at optical wavelengths (e.g. Bernardi et al. 2003a) and that produced by the dissipational simulations. Black hole feedback causes the full-model remnants to be slightly larger than the dissipational model remnants as feedback-driven winds remove gaseous material from the innermost regions of the remnants that would otherwise contribute to the central stellar content. Figure 5 shows the full-model remnants produced by mergers appropriate for various redshifts on nearly radial, parabolic orbits. The resultant FP relation is roughly independent of the redshift scalings of the progenitor systems and the location of remnants within the FP is fairly insensitive to the orientations of the disks (purple pentagons, see Table 2 and orbital configuration (orange hexagons, see Table 2). As these simulations indicate, varying the total angular momentum by changing either the orientation of the disks (spin) or the pericentric passage distance (orbital) does not strongly influence the FP of the remnants. Typically the angular momentum of the orbit only influences

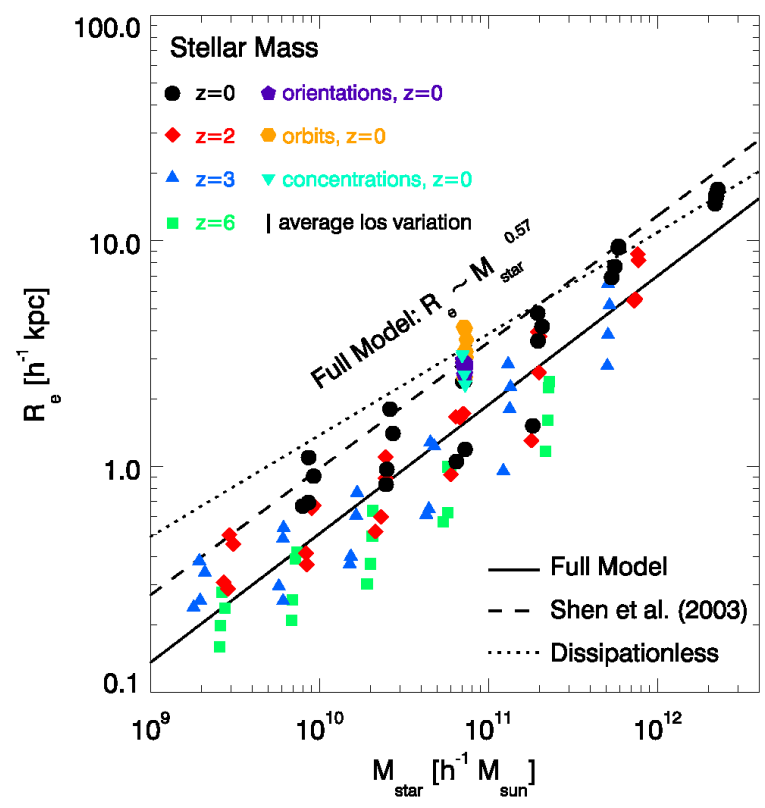

FIG. 6.- Effective radius $R_{\mathrm{e}}-$ stellar mass $M_{\star}$ relation produced by the merging of gas-rich disk galaxies with dark matter halos, star formation, supernova feedback, and a prescription for feedback from accreting supermassive black holes. Shown are remnants produced by mergers appropriate for redshifts $z=0$ (black circles), $z=2$ (red diamonds), $z=3$ (blue triangles), and $z=6$ (green squares) with nearly radial, parabolic orbits. Also plotted are remnants produced by varying the system angular momentum through the initial disk orientation (purple pentagons) and pericentric passage distances (orange hexagons) for a single pair of progenitor models, which produces a spread in the remnant effective radius. The merging of pure disk galaxies using our full physical model produces a $R_{\mathrm{e}}-M_{\star}$ relation roughly parallel to that measured for massive galaxies in the Sloan Digital Sky Survey (Shen et al. 2003), though with an offset. The resultant $R_{\mathrm{e}}-M_{\star}$ relation shifts downward with the redshift of the progenitor systems as the remnants decrease with size. For comparison, the best least-squares fit to the $R_{\mathrm{e}}-M_{\star}$ relation delineated by these remnants (solid line), as well as the dissipationless disk model $R_{\mathrm{e}}-M_{\star}$ relation (dotted line) and the Shen et al. (2003) relation (dashed line) are plotted. Also shown is the mean deviation induced by line-of-sight variations in projected quantities for a given remnant (detached error bars).

the effective radii of the remnants, with the velocity dispersion and surface mass density changing in a compensating fashion to maintain the FP scalings. In addition, varying the concentration of the dark matter halo by a factor of $\sim 2.5$ does little to change the FP scalings (cyan triangles).

The influence of angular momentum on the remnant properties is plainly visible in the $R_{\mathrm{e}}-M_{\star}$ relation for the full-model simulations (Figure 6). As is evident in the FP scalings, simulated mergers appropriate for redshifts $z=0-6$ produce results very similar to the dissipational runs with a comparable $R_{\mathrm{e}}-M_{\star}$ scaling $(\mu=0.57)$ and slightly larger remnants. However, varying the angular momentum of the merging system through changing the progenitor disk orientations (purple pentagons) and pericentric passage distances for several orientations (orange hexagons) can as much as double the average effective radius of the remnant produced by the same progenitors. The dark matter halo concentration also has a 


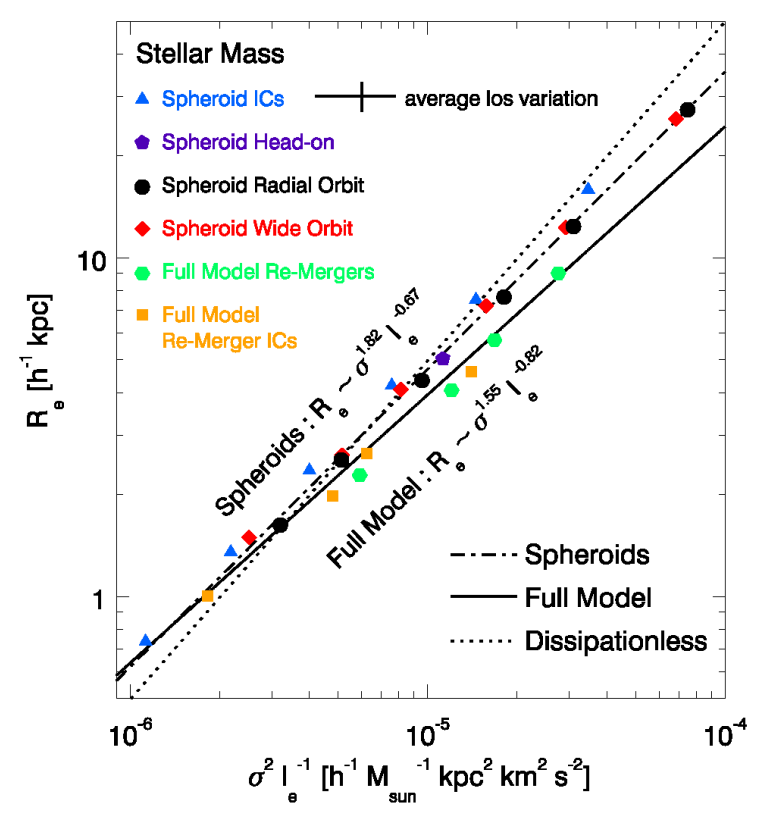

FIG. 7.- Fundamental Plane (FP) relation produced by the merging of spheroidal galaxy models. Using Hernquist (1990) stellar spheroids models with dark matter halos as initial conditions (blue triangles), the remnants produced by dissipationless spheroid mergers appropriate for redshift $z=0$, with nearly radial, parabolic orbits (black circles) and wide, elliptical orbits with circularity $\epsilon=0.5$ (red diamonds) are calculated. The remnants of spheroidal mergers produce a FP relation similar to their progenitor systems, roughly independent of the orbital energy or angular momentum. Also shown are select spheroidal remnants from gas-rich disk galaxy merger simulations that include star formation, supernova feedback, and a prescription for feedback from accreting supermassive black holes (orange squares). The disk galaxy merger remnants occupy a FP relation similar to that observed in infrared observations (solid line). The re-merging of these disk galaxy remnants on nearly radial, parabolic orbits (green circles), further demonstrates that the merging of spheroidal remnants produces a FP similar to that occupied by the progenitor systems (e.g. Capelato et al. 1995; Dantas et al. 2003; Nipoti et al. 2003; Bovlan-Kolchin et al. 2005). For comparison, the best least-squares fit to the FP delineated by the dissipationless disk merger remnants (dotted line) and spheroidal merger remnants (dash-dotted line) is plotted. Also shown is the mean deviation induced by line-of-sight variations in projected quantities for a given remnant (detached error bars).

noticeable effect on the galaxy size (cyan triangles). We reiterate that these changes in the effective radius only induce a spread parallel to the FP (see above). Moreover, as is the case for the dissipational simulations without black hole growth, the $R_{\mathrm{e}}-M_{\star}$ relation for the full model remnants would only benefit from subsequent re-merging or more cosmologically representative orbits by increasing $R_{\mathrm{e}}$.

\subsection{Spheroid Simulations}

As demonstrated in $4.1-4.3$ the merging of dissipationless disk galaxies produces a FP with almost no tilt relative to the virial plane and a $R_{\mathrm{e}}-M_{\star}$ relation that is shallower than observed, while gas-rich disk galaxies yield FP relations with substantial tilt and reasonable $R_{\mathrm{e}}-M_{\star}$ scalings. A natural extension of these calculations is to test whether the subsequent merging of the remnants or model galaxies similar in struc-

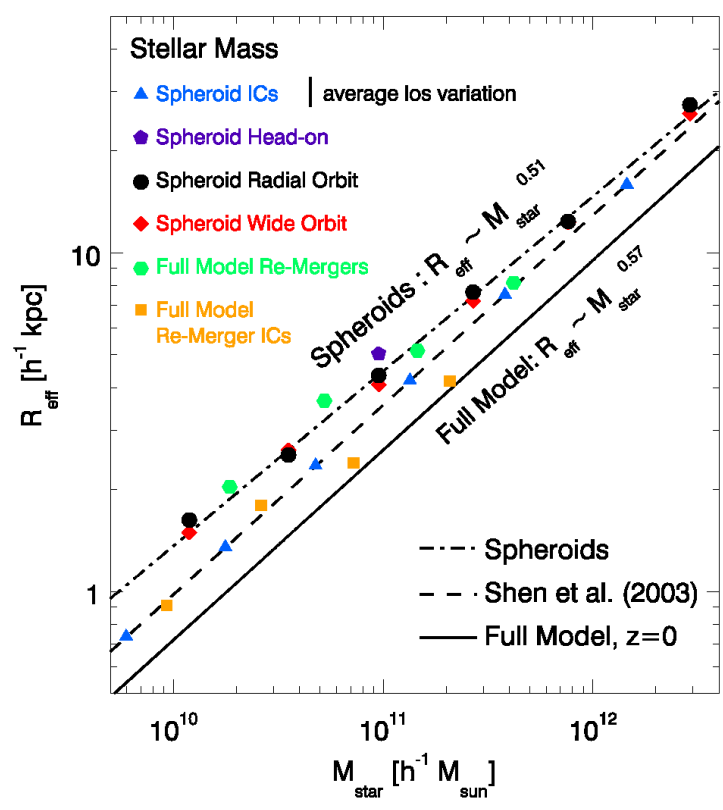

FIG. 8.- Effective radius $R_{\mathrm{e}}-$ stellar mass $M_{\star}$ relations produced by the merging of spheroidal galaxy models. The mergers of Hernquist (1990) stellar spheroids initial conditions with dark matter halos (blue triangles), initialized to the Shen et al. (2003) relation (dashed line), with nearly radial, parabolic orbits (black circles) and wide, elliptical orbits with circularity $\epsilon=0.5$ (red diamonds) are simulated. The remnants of spheroidal mergers produce a $R_{\mathrm{e}}-M_{\star}$ relation slightly shallower than the observed relation, roughly independent of the orbital energy or angular momentum. Also shown are spheroidal remnants from gas-rich disk galaxy merger simulations that include star formation, supernova feedback, and a prescription for feedback from accreting supermassive black holes (orange squares). The disk galaxy merger remnants occupy a $R_{\mathrm{e}}-M_{\star}$ relation with the same scaling as observed in SDSS (dashed line). The re-merging of these disk galaxy merger remnants on nearly radial, parabolic orbits (green circles) produces a similar $R_{\mathrm{e}}-M_{\star}$ relation as the dissipationless merging of spheroidal galaxies. For comparison, the best least-squares fit to the $R_{\mathrm{e}}-M_{\star}$ relation delineated by the dissipationless spheroidal merger remnants (dashed-dotted line), the Shen et al. (2003) relation, and the relation produced by simulations using the full physical model (solid line) are plotted. Also shown is the mean deviation induced by line-of-sight variations in projected quantities for a given remnant (detached error bars).

ture to the remnants preserves the FP and $R_{\mathrm{e}}-M_{\star}$ relations produced by the first generation of mergers. The spheroidal galaxy model progenitors described in $\$ 3.4$ that are initialized to obey the observed Shen et al. (2003) $R_{\mathrm{e}}-M_{\star}$ relation delineate a tight FP relation (blue triangles, Figure 7). Merging these spheroidal galaxy models on either nearly-radial parabolic orbits (red diamonds), wide slightly-bound orbits (black circles), or in an exactly head-on encounter (purple pentagon) produces a similar tight FP relation, only slightly tilted relative to the progenitor FP scalings. As described previously, altering the angular momentum of the orbits has the effect of increasing the effective radii of the remnants but does not strongly affect the FP relation as the velocity dispersion and surface mass density compensate to roughly maintain the progenitor FP scalings.

A similar result is obtained by re-merging remnants from the full-model simulations. The FP delineated by the full- 
model remnants (Figure 7] solid line) is roughly recovered by re-merging the disk galaxy remnants, which demonstrates that the approximate preservation of the FP relation holds for relations instilled by hand (as for spheroidal model galaxies) and those generated self-consistently through the merging process (as for the full-model merger remnants). The re-merger remnants are consistently larger in effective radii when compared with their progenitors, also in agreement with the spheroidal galaxy model mergers and previous work (e.g. Boylan-Kolchin et al. 2005).

The behavior of the $R_{\mathrm{e}}-M_{\star}$ relation under merging demonstrates the increase of $R_{\mathrm{e}}$ of spheroidal and re-merger remnants relative to their progenitor properties. As noted previously (Barnes 1992; Hernquist et al. 1993; Cole et al. 2000; Nipoti et al. 2003; Shen et al. 2003; Boylan-Kolchin et al. 2005), the effective radius of the spheroidal merger remnants approximately doubles in an equal-mass merger and will introduce scatter parallel to the $R_{\mathrm{e}}$-axis. However, as is apparent from Figure 8 the $R_{\mathrm{e}}-M_{\star}$ relation only moderately changes as a mass-sequence (e.g. $\mu=0.56$ for spheroidal progenitors, $\mu=0.52$ for spheroidal merger remnants). Re-mergers of disk galaxy remnants produce a similar change in the $R_{\mathrm{e}}-M_{\star}$ relation. A dissipationless merger between spheroids then is expected to introduce scatter into the $R_{\mathrm{e}}-M_{\star}$ relation but does not completely destroy the $R_{\mathrm{e}}-M_{\star}$ correlation of the progenitors. The presence of a well-defined $R_{\mathrm{e}}-M_{\star}$ correlation at $z=0$ is therefore not a strong constraint on the dissipationless merging of spheroids (c.f. Shen et al. 2003), although substantial additional merging beyond the recently inferred $0.5-1$ dissipationless mergers at redshifts $z<1$ typical for spheroidal galaxies (Bell et al. 2005; van Dokkum 2005) could possibly induce noticeable scatter into the $R_{\mathrm{e}}-M_{\star}$ relation beyond that reported by Shen et al. (2003).

We note briefly here that the maintenance of a $R_{\mathrm{e}}-M_{\star}$ relation with $\mu \approx 0.5$ also will keep the $M_{\mathrm{BH}}-\sigma$ relation scaling of $M_{\mathrm{BH}} \propto \sigma^{4}$, while possibly inducing some additional scatter owing to a shift in the normalization toward lower velocity dispersions at a given black hole mass. The velocity dispersion of a spheroidal galaxy is roughly

$$
\sigma^{2} \propto \frac{M_{\star}}{R_{\mathrm{e}}} .
$$

The maintenance of the $R_{\mathrm{e}}-M_{\star}$ relation with $\mu \approx 0.52$ after re-merging and the assumption that the stellar mass $M_{\star}$ and black hole mass $M_{\mathrm{BH}}$ both double then implies

$$
M_{\mathrm{BH}} \propto \sigma^{4.2}
$$

which is approximately the observed $M_{\mathrm{BH}}-\sigma$ scaling at $z=0$. Correspondingly, the $M_{\mathrm{BH}}-\sigma$ relation of the re-merger remnants that include black holes (see Figure 9) does not differ strongly from the $M_{\mathrm{BH}}-\sigma$ relation delineated by their progenitor systems. While $\sigma$ does not increase dramatically enough to perfectly preserve the observed relation (Nipoti et al. 2003), the measured $R_{\mathrm{e}}-M_{\star}$ relation of the remerger remnants provides a preliminary explanation for the rough maintenance of the $M_{\mathrm{BH}}-\sigma$ relation through infrequent dissipationless mergers. We also note that if dissipationless major merging happens more frequently for more massive systems, then the $M_{\mathrm{BH}}-\sigma$ relation may also steepen relative to the Tremaine et al. (2002) best-fit relation at higher masses. A signature of such dynamical reprocessing may appear as a mass-dependent scatter in the local $M_{\mathrm{BH}}-\sigma$ relation (Robertson et al. 2005b).

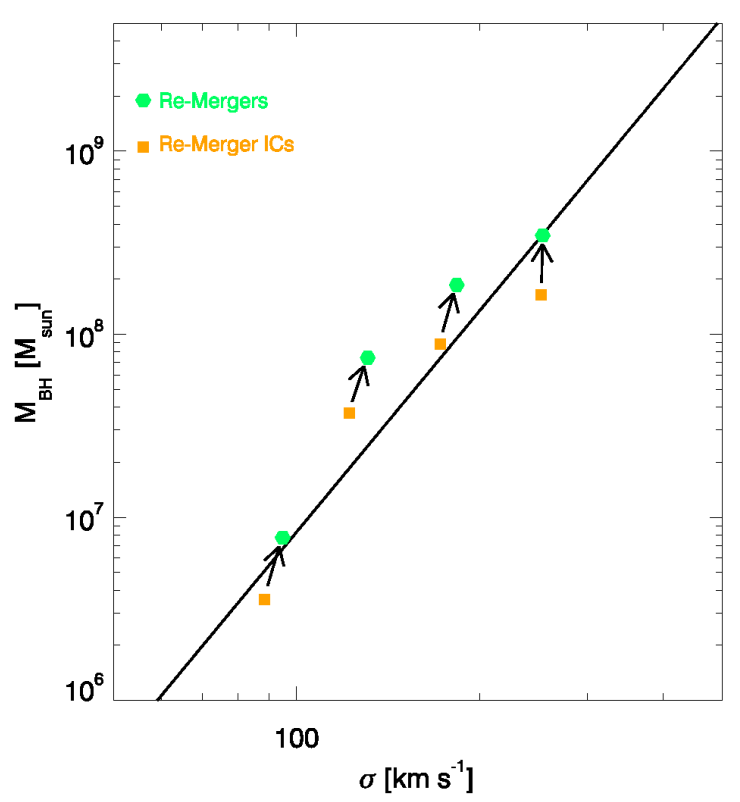

FIG. 9.- Black hole mass $M_{\mathrm{BH}}$ - stellar velocity dispersion relation $\sigma$ relation measurements for galaxies produced by the re-merging of disk galaxy merger remnants that obey the local $M_{\mathrm{BH}}-\sigma$ relation (e.g., Tremaine et al. 2002, solid line). While the number of re-mergers examined is limited, a single generation of dissipationless mergers after the initial formative gas-rich mergers that generate the $M_{\mathrm{BH}}-\sigma$ relation is not expected to strongly alter the observed $M_{\mathrm{BH}}-\sigma$ relation but may be a source of scatter.

\section{THE IMPACT OF PROGENITOR GAS FRACTION ON MERGER REMNANT PROPERTIES}

The results presented in $\$ 4$ demonstrate that the merger of gas-rich systems produces a substantially different fundamental plane scaling than the virial scalings obtained by the merging of dissipationless disk galaxies. However, the origin of the FP tilt measured for the simulations that include the effects of dissipational physics has not yet been illuminated. To better demonstrate that dissipational physics does indeed produce a FP tilt for sufficiently gas-rich systems and to demonstrate the manner by which gas dissipation gives rise to FP tilt, we now present the results of a sequence of dissipational model mergers in which the gas fraction $f_{\text {gas }}$ of the progenitor disks has been varied systematically from $f_{\text {gas }}=0.01$ to $f_{\text {gas }}=0.4$. The details of these simulations are described in $\$ 3.2$ and listed in Table 1

\subsection{Fundamental Scaling Relations}

The Fundamental Plane relation produced by the merging of disk galaxies models with dissipation as a function of progenitor gas content is shown in Figure 10 The remnants produced by progenitor systems with various gas fractions, $f_{\text {gas }}$ are plotted. The impact of dissipation on the FP tilt of remnants exhibits a behavior where for remnants of progenitors with $f_{\text {gas }}<0.2$ little FP tilt is induced by gas dissipation while for remnants of progenitors with $f_{\text {gas }}>0.2$ substantial FP tilt is induced (see Table 3 for the best-fit scalings). The nearlydissipationless simulations $\left(f_{\text {gas }}<0.1\right)$ produce FP scalings similar to the virial scalings $(\lambda=0.92-0.97)$, while the gasrich mergers $\left(f_{\text {gas }}=0.4\right)$ produce a substantial tilt $(\lambda=0.83)$. 
The $R_{\mathrm{e}}-M_{\star}$ relation of the remnants also displays a behavior where above a critical gas fraction $f_{\text {gas }} \approx 0.3$ dissipation is important for determining the remnant properties. Below $f_{\mathrm{gas}} \approx 0.2$ the $R_{\mathrm{e}}-M_{\star}$ relation is shallower than the observed Shen et al. (2003) relation and is similar to the $R_{\mathrm{e}}-M_{\star}$ relation produced by dissipationless merging of purely stellar disks. As the gas fraction of the progenitor systems increases beyond $f_{\text {gas }}=0.2$, the dissipational effects of gas cooling and star formation produce smaller remnant effective radii and a steeper $R_{\mathrm{e}}-M_{\star}$ relation (see Figure 11). The decrease in effective radius is most dramatic in lower-mass systems, where $R_{\mathrm{e}}$ can decrease by as much as $50 \%$. The tilt induced in the FP relation with progenitor gas fraction by the increasing importance of gas dissipation appears closely related to this massdependent decrease in effective radius, as the velocity dispersion and surface mass density adjust with the change in effective radius to produce FP tilt as the dissipative effects increase. We note here that the equation of state of the ISM also influences the effective radii, with the less-pressurized ISM model leading to smaller remnants as dissipation becomes more efficient with decreasing ISM pressurization.

\subsection{Phase-Space Density}

While the behavior of the fundamental scaling relations of the merger remnants as a function of progenitor gas fractions demonstrates that dissipation can induce FP tilt by steepening the $R_{\mathrm{e}}-M_{\star}$ relation, these structural parameter correlations reflect the composition of more fundamental properties of the remnants. One such property is the fine-grained phase-space density $f(x, v)$ at the spatial coordinate $x$ and velocity coordinate $v$. The quantity $f(x, v) \mathrm{d} x \mathrm{~d} v$ describes the probability of a stellar mass element $\mathrm{d} M_{\star}$ being located within the differential phase-space volume $\mathrm{d} x \mathrm{~d} v$ centered at coordinates $(x, v)$. For a purely collisionless system, $f(x, v)$ satisfies the Boltzmann equation

$$
\frac{\partial f}{\partial t}+\mathbf{v} \bullet \nabla f-\nabla \Phi \bullet \frac{\partial f}{\partial \mathbf{v}}=0 .
$$

Also known as the Vlasov formula, Equation (19) describes the detailed conservation of the phase-space density as an incompressible flow under the influence of a smoothly varying potential $\Phi(x, t)$ and represents the 1-particle limit of Liouville's theorem. The underlying phase-space structure of a dissipationless system is then a conserved, fundamental quantity.

Although the fine-grained phase-space density of stars is a basic physical property of a galaxy, the finite numerical resolution of simulations limits the measurable phase-space density to the quantity $\bar{f}(x, v) \Delta x \Delta v$ that represents the probability of a macroscopic stellar mass element $\Delta M_{\star}$ being located within the macroscopic phase-space volume $\Delta x \Delta v$ centered at $(x, v)$. The quantity $\bar{f}$ is commonly referred to as the coarsegrained distribution function (e.g. Binney \& Tremaine 1987). While $\bar{f}(x, v)$ can be measured from the simulations by gridding the stellar particles into $\Delta x \Delta v$ volumes, a well-sampled $\bar{f}(x, v)$ requires a crude $\Delta x \Delta v$ binning. Instead, one may adopt the approach of Hernquist et al. (1993) and measure the coarse-grained phase-space density $\bar{f}(E)$ averaged over hypersurfaces with energy $E$ under the assumptions of sphericity and isotropy. With these approximations, the fine grained phase-space density $f$ is a function of energy and given a model of a galaxy that provides the density of states $g(E)$, the function describing the phase-space volume of energy hypersurfaces, one may relate the mass-energy distribution of stars

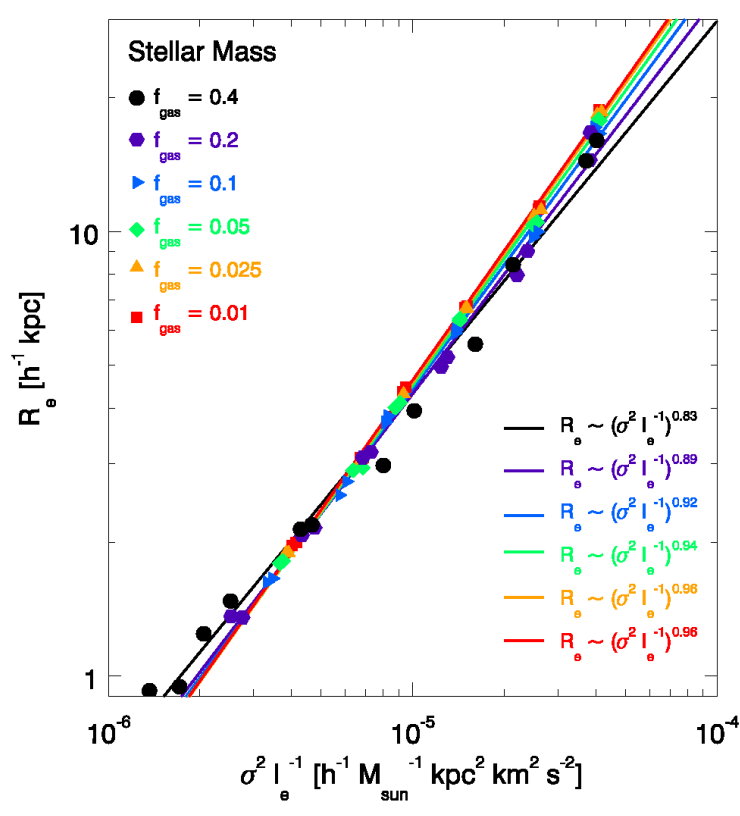

FIG. 10.- Fundamental Plane (FP) relation produced by the merging of disk galaxies with dark matter halos, star formation and supernova feedback as a function of progenitor gas content. The galaxy models are appropriate for $z=0$ and are merged on nearly radial, parabolic orbits. Shown are remnants produced by progenitor systems with gas fraction $f_{\text {gas }}=0.01$ (red squares and line), $f_{\text {gas }}=0.025$ (yellow triangles and line), $f_{\text {gas }}=0.04$ (green diamonds and line), $f_{\text {gas }}=0.1$ (blue triangles and line), $f_{\text {gas }}=0.2$ (purple hexagons and line), and $f_{\text {gas }}=0.4$ (black circles and line). As the gas fraction of the progenitor systems increases past $f_{\text {gas }}=20 \%$, the dissipational effects of gas cooling and star formation induce a substantial tilt in the resulting FP relation. Nearly dissipationless progenitor $\left(f_{\text {gas }}<20 \%\right)$ produce FP relations roughly parallel to the plane delineated by the virial relation, while for systems where gas dissipation is important the resultant FP is similar to the observed infrared FP (Pahre et al. 1998b). The increasing tilt is generated through a decrease in the remnant effective radius with increasing gas content and the corresponding change in the central stellar mass fraction (see also Figures 11- 15 .

$\mathrm{d} M_{\star} / \mathrm{d} E$ to the fine-grained phase-space density $f(E)$ as

$$
\frac{\mathrm{d} M_{\star}}{\mathrm{d} E}=f(E) g(E) .
$$

A coarse grained distribution function $\bar{f}(E)$ can be measured from the simulations through a histogram of stellar particle energies $\Delta M_{\star} / \Delta E$ as

$$
\bar{f}(E)=\frac{1}{g(E)} \frac{\Delta M_{\star}}{\Delta E} .
$$

As Hernquist et al. (1993) demonstrate, the coarse-grained $\bar{f}(E)$ provides a reliable estimate of the fine-grained $f(E)$ for numerical models of isotropic, spherical galaxies. An even more precisely measurable quantity is the cumulative distribution function

$$
s(f)=\frac{1}{M_{\star}} \int_{E_{0}}^{E(f)} \frac{\mathrm{d} M_{\star}}{\mathrm{d} E} \mathrm{~d} E,
$$

where $E_{0}$ is the central binding energy. The cumulative distribution function $s(f)$ provides the fraction of stellar mass 


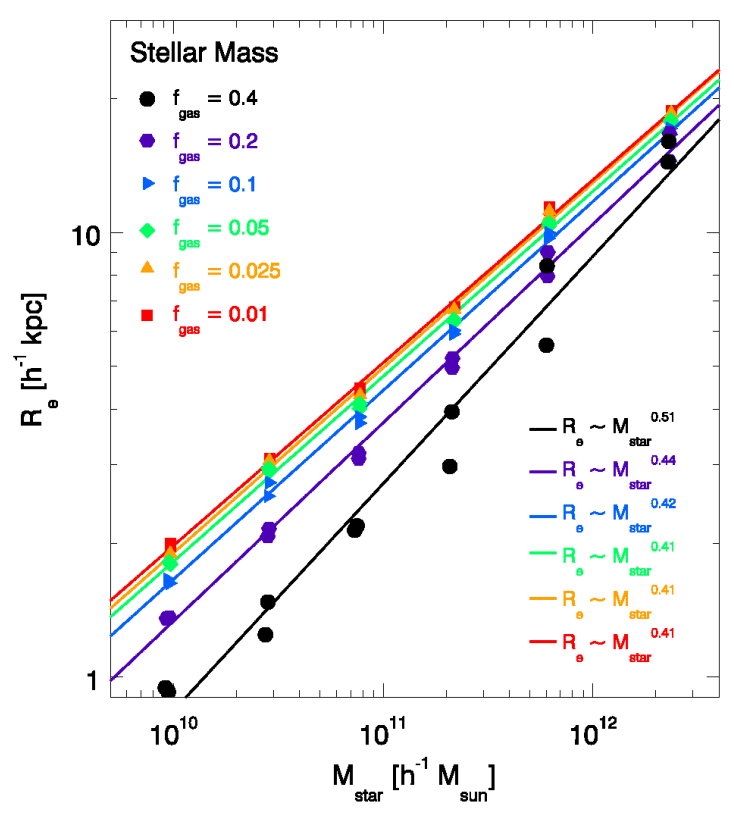

FIG. 11.- Effective radius $R_{\mathrm{e}}-$ stellar mass $M_{\star}$ relation produced by the merging of gas-rich disk galaxies with dark matter halos, star formation and supernova feedback as a function of progenitor gas content. The galaxy models are appropriate for $z=0$ and are merged on nearly radial, parabolic orbits. Shown are remnants produced by progenitor systems with gas fraction $f_{\text {gas }}=0.01$ (red squares and line), $f_{\text {gas }}=0.025$ (yellow triangles and line), $f_{\text {gas }}=0.04$ (green diamonds and line), $f_{\text {gas }}=0.1$ (blue triangles and line), $f_{\text {gas }}=0.2$ (purple hexagons and line), and $f_{\text {gas }}=0.4$ (black circles and line). As the gas fraction of the progenitor systems increases, the dissipational effects of gas cooling and star formation produce smaller remnant effective radii and a corresponding change in the central stellar mass fraction (see also Figures 10 14 and 15. These dissipational effects generate a substantial tilt in the Fundamental Plane above a gas fraction of roughly $f_{\text {gas }}>30 \%$.

at phase-space densities above $f$, which is equivalent to the fraction of stars more tightly bound than energy $E(f)$ when averaged over energy hypersurfaces. For a binned energy histogram $\Delta M_{\star} / \Delta E$ measured from the simulations, a cumulative coarse grained distribution function $s(\bar{f})$ can be calculated as

$$
s(\bar{f})=\frac{1}{M_{\star}} \sum_{i=j_{E_{0}}}^{j_{E(\bar{f})}} \frac{\left(\Delta M_{\star}\right)_{i}}{\Delta E} \Delta E,
$$

where $\left(\Delta M_{\star}\right)_{i}$ is the stellar mass with energy between $E_{i}$ and $E_{i}+\Delta E, j_{E_{0}}=0$ is the index of the energy bin with $E=E_{0}$ and $j_{E(\bar{f})}$ is the index of the energy bin with energy $E=E(\bar{f})$. The cumulative energy distribution $s(E)$ can be measured directly from the simulations, independent of any assumed model for $g(E)$. The choice of galaxy model then simply correlates $\vec{f}$ with an energy $E$.

To connect a coarse-grained phase-space density $\bar{f}$ with an energy $E$, we adopt an isotropic, stellar Hernquist (1990) spheroid embedded within a larger isotropic, dark matter Hernquist (1990) spheroid (hereafter the HH model). Ciotti (1996) analytically calculated both the distribution function $f(E)$ and density of states $g(E)$ for such a system, which serves as a convenient galaxy model for the remnants and pro-

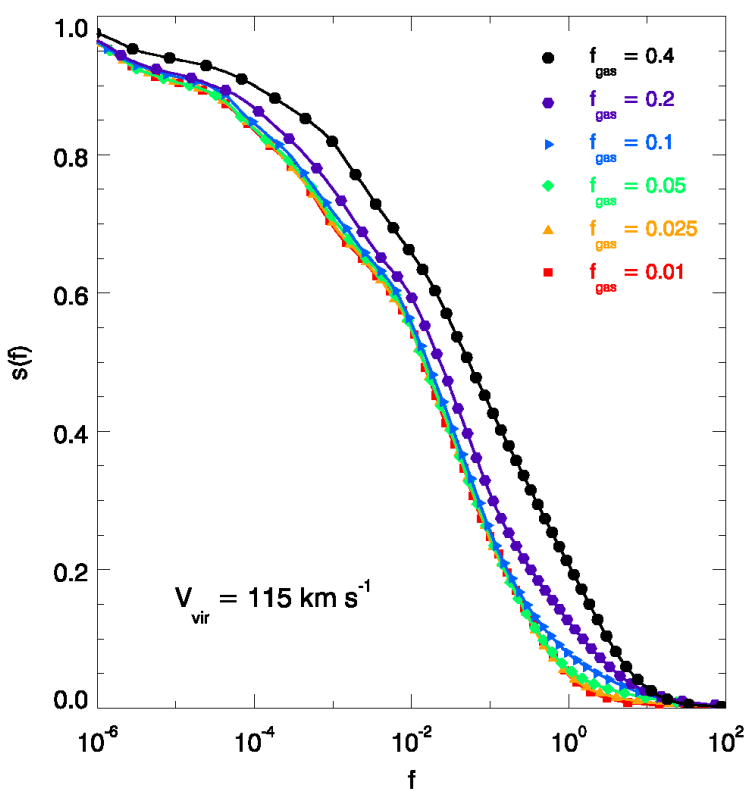

FIG. 12.- Cumulative coarse-grained distribution function $s(\bar{f})$ of a remnant from the equal-mass merger of progenitors with $V_{\text {vir }}=115$ $\mathrm{km} \mathrm{s}^{-1}$ as a function of progenitor gas fraction. Shown is the fraction of remnant stellar mass with phase-space densities greater than $\bar{f}$ for mergers with $f_{\text {gas }}=0.01$ (red line), $f_{\text {gas }}=0.025$ (orange line), $f_{\text {gas }}=0.05$ (green line), $f_{\text {gas }}=0.1$ (blue line), $f_{\text {gas }}=0.2$ (purple line), and $f_{\text {gas }}=0.4$ (black line). For gas fractions $f_{\text {gas }}<0.2$, the phasespace density distributions of the remnants do not change appreciably; the binding energies of the stars do not increase greatly relative to those in a collisionless merger as dissipation is not efficient at altering the structure of the galaxy. For gas fraction $f_{\text {gas }}>0.2$, the phase-space density distribution of the remnant shifts toward higher values and more strongly-bound energy hypersurfaces. For low mass ellipticals the phase-space density of remnants is then increased in gas-rich $f_{\text {gas }}>30 \%$ mergers where dissipation is important for the remnant properties.

vides a conversion between binding energy and phase-space density for a set of remnant scale radii $a_{\star}$ and $a_{\mathrm{DM}}$ and stellar and dark matter masses. The scale radii of the remnants can be related to the effective radius and reliably estimated from the particle distributions (e.g. \$3.5, while the mass of the stellar and dark matter components are tracked explicitly. However, as the Hernquist (1990) model may not perfectly reflect the stellar distribution, the potential well depth at the center of the merger remnant often exceeds the expected potential minimum for the $\mathrm{HH}$ model. In this case we find the effective $a_{\star}$ for the HH system that produces the correct minimum potential and use this model to calculate $f(E)$ and $g(E)$. As the functional form of $f(E)$ for the $\mathrm{HH}$ model depends only on the ratios $M_{\star} / M_{\mathrm{DM}}$ and $a_{\star} / a_{\mathrm{DM}}$ this technique for measuring $s(\bar{f})$ is fairly insensitive to the manner in which we measure galaxy properties. Once violent relaxation reduces the timedependence of the galaxy potential during a dissipationless merger, the form of $s(\bar{f})$ will be fixed, while for gaseous mergers, the form of $s(\bar{f})$ will stabilize after the additional process of star formation becomes relatively unimportant. A comparison of the $s(\bar{f})$ resulting from strongly dissipative mergers with the $s(\bar{f})$ of dissipationless remnants will indicate the impact of dissipation on the final galaxy phase-space structure. 


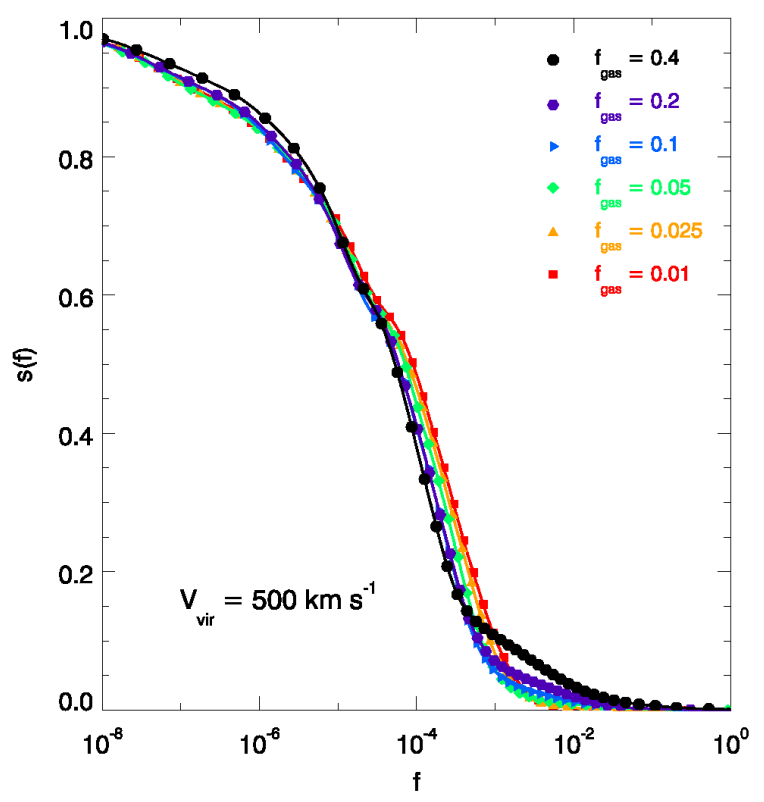

FIG. 13.- Cumulative coarse-grained distribution function $s(\bar{f})$ of a remnant from the equal-mass merger of progenitors with $V_{\text {vir }}=500$ $\mathrm{km} \mathrm{s}^{-1}$ as a function of progenitor gas fraction. Shown is the fraction $s(\bar{f})$ of remnant stellar mass with phase-space densities greater than $\bar{f}$ for mergers with $f_{\text {gas }}=0.01$ (red line), $f_{\text {gas }}=0.025$ (orange line), $f_{\text {gas }}=0.05$ (green line), $f_{\text {gas }}=0.1$ (blue line), $f_{\text {gas }}=0.2$ (purple line), and $f_{\text {gas }}=0.4$ (black line). For gas fractions $f_{\text {gas }}<0.2$, the phasespace density distributions of the remnants do not change appreciably; the binding energies of the stars do not increase greatly relative to those in a collisionless merger as dissipation is not efficient at altering the structure of the galaxy. For a gas fraction $f_{\text {gas }}>0.2$ the phase-space density distribution of the remnant increases at moderate phase-space densities relative to the dissipationless remnant, but not dramatically. The comparatively small shift in $s(\bar{f})$ for massive ellipticals formed in gas-rich mergers relative to the larger resultant shift in low mass ellipticals results in a mass-dependent trend in $M_{\text {total }} / M_{\star}$ (see Figures 14-15.

Figure 12 shows the cumulative coarse-grained distribution function $s(\bar{f})$ of a remnant from the equal-mass merger of progenitors with $V_{\text {vir }}=115 \mathrm{~km} \mathrm{~s}^{-1}$ as a function of progenitor gas fraction. The measured $s(\bar{f})$ for gas fractions below $f_{\text {gas }}<0.2$ remains roughly constant. The binding energies of the stars are not strongly affected by the weak influence of dissipation on the galaxy structure for low gas fraction merger, but as the progenitor gas fractions increase beyond $f_{\text {gas }}>0.2$ the phase-space density distribution of the remnant shifts toward higher values and more strongly-bound energy hypersurfaces. Strongly dissipational mergers clearly lead to higher phase-space densities in low mass elliptical galaxy remnants. We note here that the shapes of the cumulative phase-space density as a function of mass and gas fraction remain very similar for either ISM pressurization we study in this work ( $q_{\mathrm{EOS}}=0.25$ and $\left.q_{\mathrm{EOS}}=1.0\right)$, except for a slight decrease in the maximum phase-space density reached in the $q_{\mathrm{EOS}}=1.0$ simulations.

For the impact of dissipation to affect the FP tilt or $R_{\mathrm{e}}-M_{\star}$ slope, the degree to which the phase-space density increases during gas-rich mergers must vary as a function of galaxy mass. Figure 13 shows the cumulative coarse grained dis-

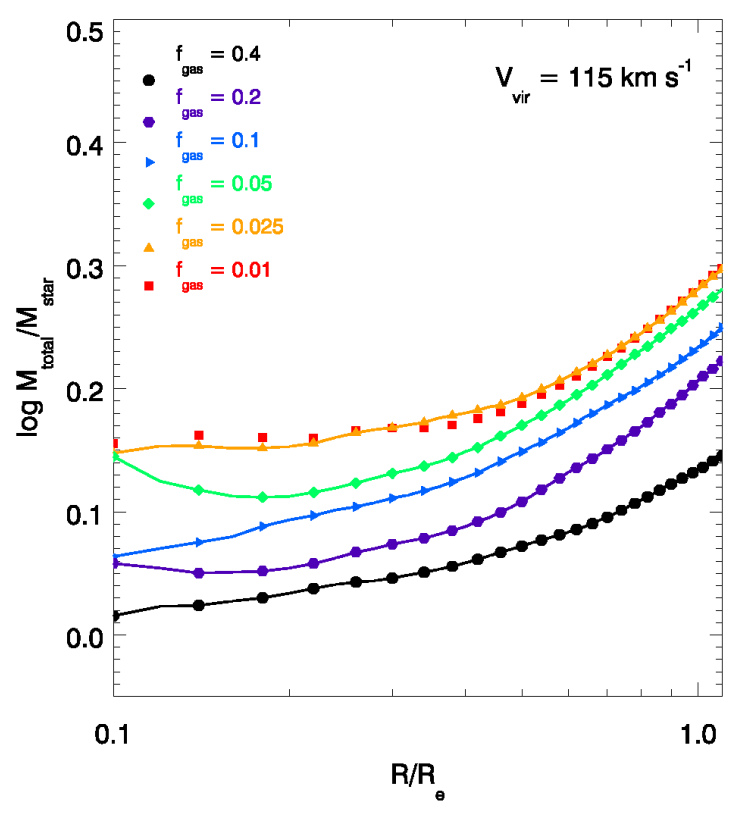

FIG. 14.- Ratio of total mass $M_{\text {total }}$ to stellar mass $M_{\star}$ within a spherical radius for the remnants of equal mass mergers of $V_{\mathrm{vir}}=115$ $\mathrm{km} \mathrm{s}^{-1}$ disk galaxies with dark matter halos, star formation and supernova feedback as a function of progenitor gas content. The galaxy models are appropriate for $z=0$ and are merged on nearly radial, parabolic orbits. Shown is $M_{\text {total }} / M_{\star}$ vs. $R / R_{\mathrm{e}}$ for remnants produced by progenitor systems with gas fraction $f_{\text {gas }}=0.01$ (red line), $f_{\text {gas }}=0.025$ (yellow line), $f_{\text {gas }}=0.04$ (green line), $f_{\text {gas }}=0.1$ (blue line), $f_{\text {gas }}=0.2$ (purple line), and $f_{\text {gas }}=0.4$ (black line). For smallmass spheroidal remnants, as the progenitor gas content is increased the stellar content substantially increases in the very central regions $\left(R<0.3 R_{\mathrm{e}}\right)$ of the galaxy. The decrease in $M_{\text {total }} / M_{\star}$ follows the corresponding decrease in $R_{\mathrm{e}}$ as star formation is concentrated in the high central gas overdensities induced by the merger.

tribution function $s(\bar{f})$ for remnants of a fiducial equal-mass merger between disk galaxies with $V_{\text {vir }}=500 \mathrm{~km} \mathrm{~s}^{-1}$ as a function of progenitor gas fraction. While the behavior for low gas fractions $f_{\text {gas }}<0.2$ is similar between low mass ellipticals (Figure 12) and massive ellipticals, the strong shift seen in the phase-space density distribution of low mass ellipticals is not measured for the more massive system. The simulations show that the impact of dissipative physics on the phase-space density of ellipticals is mass-dependent, being more important for low mass galaxies.

\subsection{Central Mass-to-Light Ratio}

With a measured mass-dependent change in phase-space density of remnants produced in gas-rich mergers, the phasespace density can be directly connected to changes in the FP tilt and $R_{\mathrm{e}}-M_{\star}$ slope induced by strongly dissipational merging. The correspondence between phase-space density $\bar{f}$ and binding energy demonstrates that the regions at the center of the galactic potential are gaining stellar mass density. The gas dissipation has a less dramatic effect on the dark matter distribution of the galaxy, and while the gas does change the dark matter profile by gravitationally dragging the dark matter inwards, the effective radius of the dark matter decreases by only $250 h^{-1} \mathrm{pc}(0.6 \%)$ between the $f_{\text {gas }}=0.1$ and $f_{\text {gas }}=0.4$ runs for $V_{\text {vir }}=115 \mathrm{~km} \mathrm{~s}^{-1}$ halos. Dissipation affects the stellar 


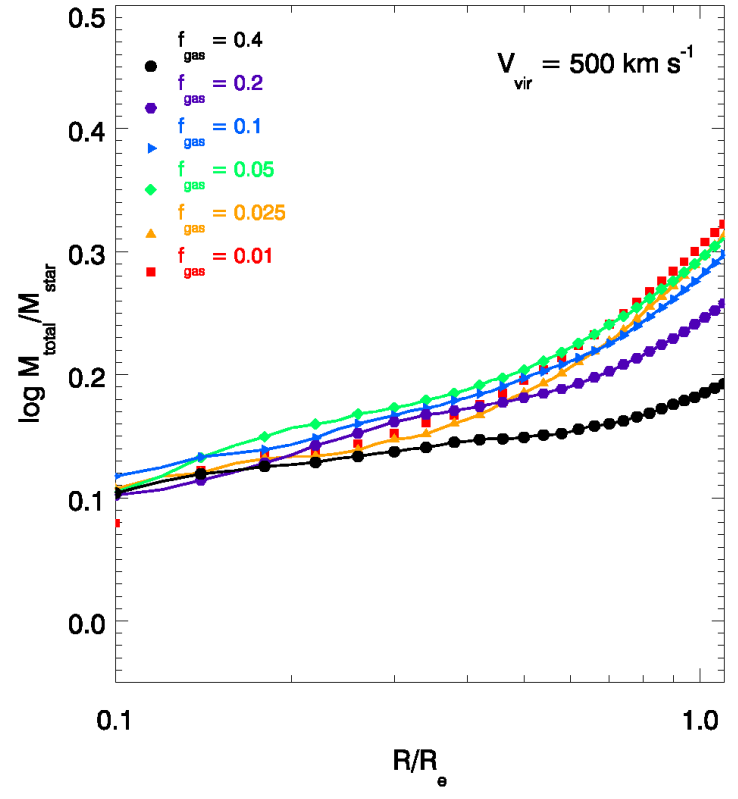

FIG. 15.- Ratio of total mass $M_{\text {total }}$ to stellar mass $M_{\star}$ with spherical radius for the remnants of equal mass mergers of $V_{\mathrm{vir}}=500 \mathrm{~km} \mathrm{~s}^{-1}$ disk galaxies with dark matter halos, star formation and supernova feedback as a function of progenitor gas content. The galaxy models are appropriate for $z=0$ and are merged on nearly radial, parabolic orbits. Shown is $M_{\text {total }} / M_{\star}$ vs. $R / R_{\mathrm{e}}$ for remnants produced by progenitor systems with gas fraction $f_{\text {gas }}=0.01$ (red line), $f_{\text {gas }}=0.025$ (yellow line), $f_{\text {gas }}=0.04$ (green line), $f_{\text {gas }}=0.1$ (blue line), $f_{\text {gas }}=0.2$ (purple line), and $f_{\text {gas }}=0.4$ (black line). For large-mass spheroidal remnants, as the progenitor gas content is increased the central stellar content begins to increase substantially after $R \gtrsim 0.3 R_{\mathrm{e}}$. The $M_{\text {total }} / M_{\star}$ value near the center is larger for massive systems than for less-massive spheroids (see Figure 14, reflecting the increasing importance of dark matter at the center of massive spheroidal galaxies.

mass much more dramatically than the dark matter mass, and the net result is a radius-dependent change in the $M_{\text {total }} / M_{\star}$ ratio as a function of progenitor gas fraction.

Figure 14 shows the ratio of total mass $M_{\text {total }}$ to stellar mass $M_{\star}$ within a spherical radius $R$ for the binary $V_{\text {vir }}=115 \mathrm{~km}$ $\mathrm{s}^{-1}$ mergers with various progenitor gas fractions, $f_{\text {gas }}$. As the effects of dissipation increase, the central regions of the lowmass remnant become progressively more baryon-dominated. The decrease in the central $M_{\text {total }} / M_{\star}$ follows the corresponding decrease in $R_{\mathrm{e}}$ as star formation is concentrated in the high central gas overdensities induced by the merger. The value of $M_{\text {total }} / M_{\star}$ does not change appreciably at $R=R_{\mathrm{e}}$ until the gas fraction reaches $f_{\text {gas }}=0.1$ and decreases dramatically as the gas fraction increases toward $f_{\text {gas }}=0.4$.

The impact of dissipation on $M_{\text {total }} / M_{\star}$ of more massive systems is less important, as expected from the behavior of the phase-space density and $R_{\mathrm{e}}$ of those systems. Figure 15 shows the ratio $M_{\text {total }} / M_{\star}$ with spherical radius $R$ for the remnants of equal mass mergers of $V_{\text {vir }}=500 \mathrm{~km} \mathrm{~s}^{-} 1$ systems with various gas fractions. For high-mass spheroidal remnants, as the progenitor gas content is increased, the stellar content begins to increase substantially at radii $R>0.3 R_{\mathrm{e}}$. The central $M_{\text {total }} / M_{\star}$ remains relatively unaffected by dissipational physics and remains roughly constant $M_{\text {total }} / M_{\star} \approx 1.25$. The

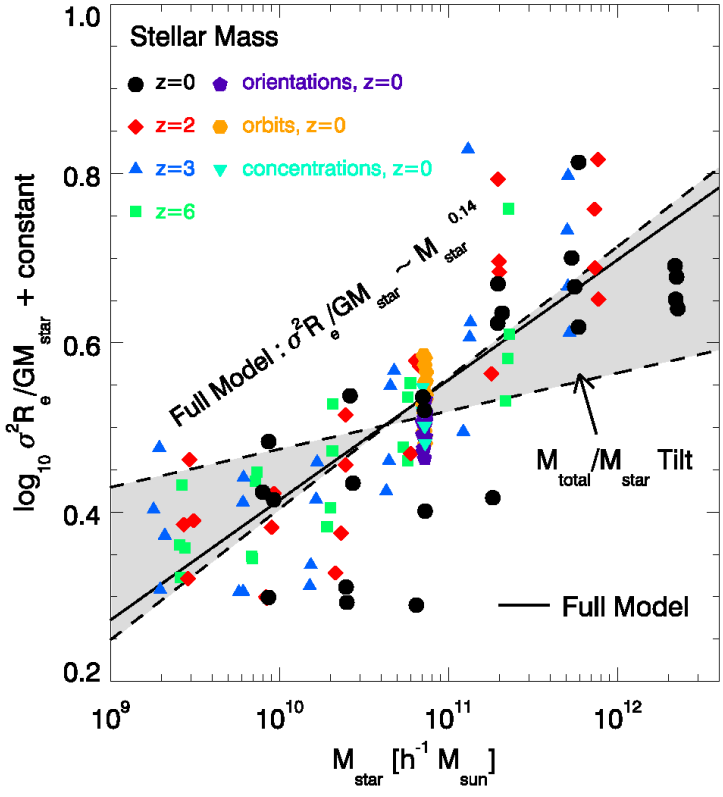

FIG. 16. - Ratio of the dynamical mass estimator $M_{\text {dyn }} \equiv \sigma^{2} R_{\mathrm{e}} / G$ to the stellar mass $M_{\star}$ in the full-model simulations as a function of stellar mass for remnants of mergers between progenitor disk galaxies appropriate for redshifts $z=0$ (black circles), $z=2$ (red diamonds), $z=3$ (blue triangles), and $z=6$ (green squares). Also shown is the measured $M_{\mathrm{dyn}} / M_{\star}$ for $z=0$ mergers with varying disk orientations (purple pentagons), orbital parameters (orange hexagons), and dark matter concentrations (cyan triangles). The best-fit trend $M_{\text {dyn }} / M_{\star} \propto M_{\star}^{0.14}$ (solid line) recovers the complete fundamental plane tilt measured for the full-model simulations in Figure 5 We also estimate the tilt contributed by variations of $M_{\text {total }} / M_{\star}$ averaged within an effective radius as a function of stellar mass measured from the remnant particle distribution (shaded area, see 5.3.1, which contributes $\approx 40-100 \%$ of the nonhomology-related FP tilt. The $M_{\text {total }} / M_{\star}$ tilt estimates shown have been renormalized by a constant to intersect with the best-fit $M_{\mathrm{dyn}} / M_{\star}$ relation at the same location.

value of $M_{\text {total }} / M_{\star}$ near the center is larger for massive systems than for less-massive spheroids (see Figure 14), reflecting the increasing importance of dark matter at the center of massive spheroidal galaxies and contributing tilt to the FP.

\subsubsection{Dynamical Mass-to-Light Ratio}

For the stellar-mass FP relation, an observationally relevant quantity is the estimator of the dynamical mass within an $R_{\mathrm{e}}$

$$
M_{\text {dyn }} \equiv k \frac{\sigma^{2} R_{\mathrm{e}}}{G}
$$

where $G$ is the gravitational constant and $k$ is a constant that depends on the structure of the galaxy $(k \approx 7.25$ for a kinematically isotropic Hernquist (1990) spheroid). The dynamical mass estimator allows us to characterize the FP tilt in terms of the observationally-accessible mass ratio

$$
\frac{M_{\text {dyn }}}{M_{\star}} \propto M_{\star}^{\gamma} .
$$

For a stellar-mass FP of the form $R_{\mathrm{e}} \propto \sigma^{\alpha} I_{\mathrm{e}}^{-\beta}$ and an $R_{\mathrm{e}}-M_{\star}$ relation of the form $R_{\mathrm{e}} \propto M_{\star}^{\mu}$, the power-law index $\gamma$ can be estimated as

$$
\gamma=\mu(2 / \alpha+1-4 \beta / \alpha)+2 \beta / \alpha-1 .
$$




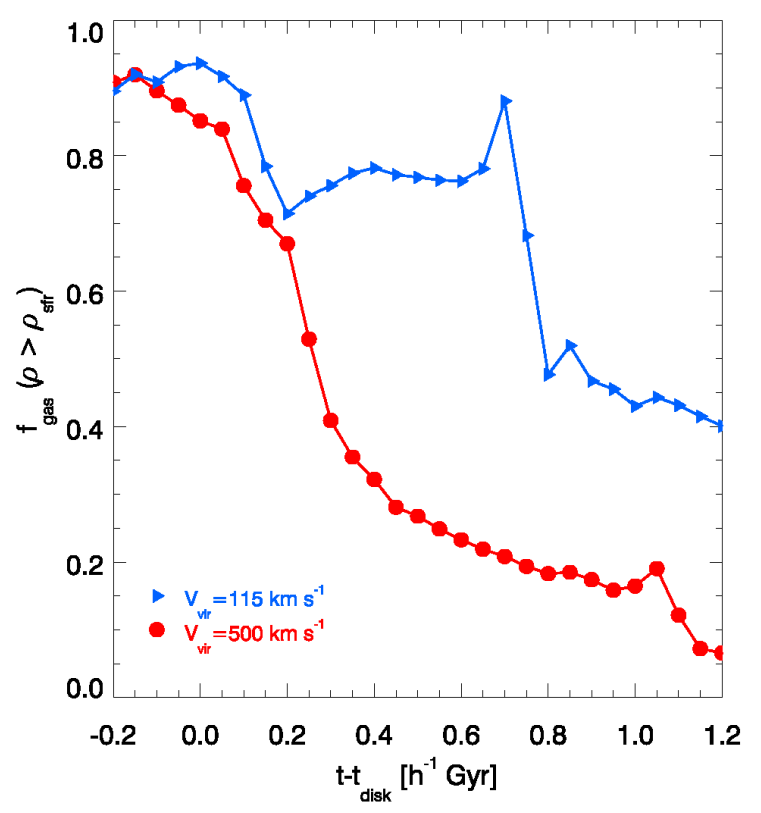

FIG. 17.- Mass fraction of star forming gas for $V_{\text {vir }}=115 \mathrm{~km} \mathrm{~s}^{-1}$ (blue triangles) and $V_{\mathrm{vir}}=500 \mathrm{~km} \mathrm{~s}^{-1}$ (red circles) progenitor merger remnants in simulations with gas fraction $f_{\text {gas }}=0.4$ as a function of time $t-t_{\text {disk }}$ since the disks first interact. The gas in the massive system is collisionally heated during the first passage and subsequently cannot cool and form stars, while gas in the small mass system can cool sufficiently to continue to form stars throughout the remainder of the merger.

For the virial scalings ( $\alpha=2, \beta=1)$, the dynamical mass estimator is proportional to the stellar mass and there is no FP tilt $(\gamma=0)$, independent of the $R_{\mathrm{e}}-M_{\star}$ relation. The results presented in $\$ 4.3$ indicate that the full-model FP $(\alpha=1.55$, $\beta=0.82$ ) is tilted relative to the virial scalings. Combined with the full-model $R_{\mathrm{e}}-M_{\star}$ relation $(\mu=0.57)$, we expect that $M_{\text {dyn }} / M_{\star} \propto M_{\star}^{0.15}$. Figure 16 shows the quantity $M_{\text {dyn }} / M_{\star}$ as a function of stellar mass for the full-model remnants from mergers of progenitor disk galaxies appropriate for redshifts $z=0-6$. While there is considerable scatter in $M_{\mathrm{dyn}} / M_{\star}$, which mostly arises from the linear dependence of $M_{\mathrm{dyn}}$ on $R_{\mathrm{e}}$, the best fit trend in the simulations has a scaling $M_{\mathrm{dyn}} / M_{\star} \propto$ $M_{\star}^{0.14}$ (solid line) that recovers essentially the complete tilt inferred for the full-model FP.

In principle, the tilt in the stellar-mass FP inferred from the dynamical mass estimator may include contributions from total mass-to-stellar mass $M_{\text {total }} / M_{\star}$ variations, kinematic nonhomologies that affect the velocity dispersion as a function of stellar mass, or other sources of nonhomology. Figure 16 shows the estimated range of tilt contributed by $M_{\text {total }} / M_{\star}$ variations with stellar mass averaged over an effective radius as measured from the particle distributions of the remnants (shaded area), bounded by least-squares fits to the $M_{\text {total }} / M_{\star}$ trend with mass minimizing with respect to either $M_{\text {total }} / M_{\star}$ or $M_{\star}$. Based on this comparison, we estimate that variations of $M_{\text {total }} / M_{\star}$ with stellar mass averaged over an effective radius contribute $\approx 40-100 \%$ of the nonhomology-related tilt in the full-model simulations.

\subsection{Dissipation vs. Galaxy Mass}

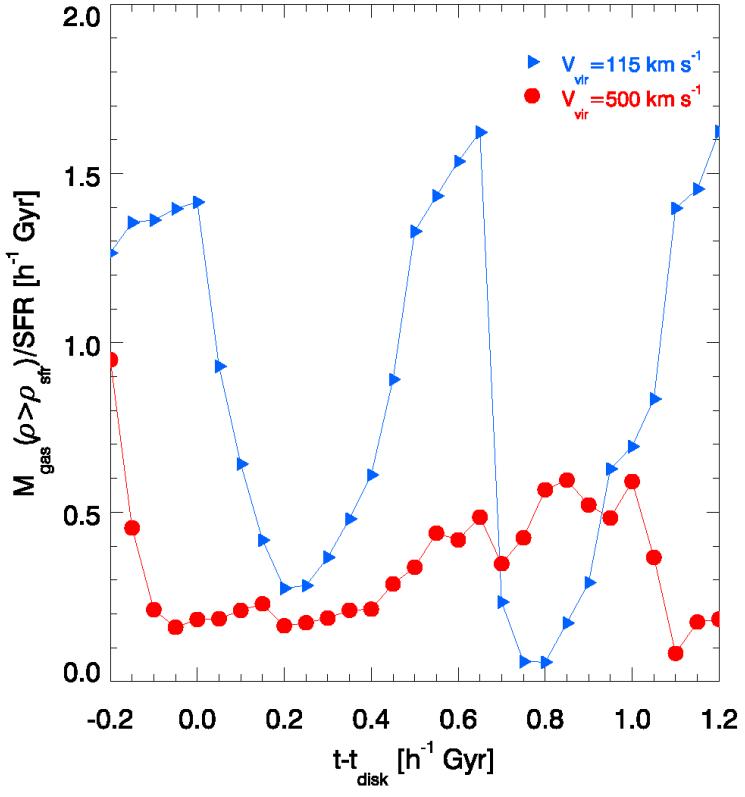

FIG. 18.- Consumption timescale of star forming gas in $V_{\text {vir }}=115$ $\mathrm{km} \mathrm{s}^{-1}$ (blue triangles) and $V_{\mathrm{vir}}=500 \mathrm{~km} \mathrm{~s}^{-1}$ (red circles) progenitor mergers measured for simulations with gas fraction $f_{\text {gas }}=0.4$ and equation of state parameter $q_{\mathrm{EOS}}=0.25$. The consumption timescale is plotted as a function of time $t-t_{\text {disk }}$ since the disks first interact and reflects the ratio of $M_{\mathrm{gas}}\left(\rho>\rho_{\mathrm{sfr}}\right) /\left(\mathrm{d} M_{\star} / \mathrm{d} t\right)$ during the simulation for gas above the density threshold for star formation. The cold gas in the massive system that is not collisionally heated by the first pericentric passage into the halo (see Figure 17 is always efficiently converted into stars. In smaller systems, the cold gas in the system is efficiently converted into stars only during times of interactions, such as pericentric passage $\left(t-t_{\text {disk }}=0.1-0.2 \mathrm{~h}^{-1} \mathrm{Gyr}\right)$ or final coalescence $\left(t-t_{\text {disk }} \approx 0.9 \mathrm{~h}^{-1} \mathrm{Gyr}\right)$, but is consumed on long timescales otherwise.

The previous sections have demonstrated that gas dissipation impacts the fundamental scaling properties of ellipticals by altering their central stellar content in a mass-dependent manner, being more important for small mass systems. We now explain how this mass-dependent impact of dissipation arises in our simulations.

Figure 17 shows the mass fraction of dense, star forming gas in merging disk galaxies as a function of the time $t-t_{\text {disk }}$ since the gaseous disks first interact for a simulation with progenitor gas fraction $f_{\text {gas }}=0.4$ and equation of state parameter $q_{\mathrm{EOS}}=0.25$. In massive systems, such as the $V_{\text {vir }}=500 \mathrm{~km}$ $\mathrm{s}^{-1}$ progenitor equal-mass merger shown here (red circles), a large fraction of the gas is collisionally heated during the first pericentric passage. This hot gas becomes X-ray luminous but cannot cool efficiently (Cox et al. 2004, 2005a), and mostly remains in the halo for the duration of the merger. The efficiency of halo gas cooling decreases with halo mass, causing more massive systems to act increasingly like dissipationless systems. In small systems (we show an equal-mass merger of $V_{\mathrm{vir}}=115 \mathrm{~km} \mathrm{~s}^{-1}$ progenitors, blue triangles), collisionally heated gas can cool efficiently and return to the disk to continuously form stars. The continued presence of star forming gas in small mass systems allows for very high phase-space density stars to form in abundance during the height of the merger, decreasing the central $M_{\text {total }} / M_{\star}$ in the final remnant. 
Although collisional heating of the gas in massive systems limits the fraction of gas dense enough to form stars, the remaining dense gas is efficiently turned into stars throughout the merger. Figure 18 shows the consumption timescale

$$
t_{\text {consumption }}=M_{\text {gas }}\left(\rho>\rho_{\mathrm{sfr}}\right)\left(\frac{\mathrm{d} M_{\star}}{\mathrm{d} t}\right)^{-1}
$$

which indicates the characteristic timescale for the mass of gas at densities above the star formation threshold $M_{\text {gas }}(\rho>$ $\rho_{\text {sfr }}$ ) to be converted into stars at the current star formation rate $\mathrm{d} M_{\star} / \mathrm{d} t$. The massive system is always efficiently converting the available gas into stars, roughly independent of the progenitor separation after first approach when tidal deformation of the disk begin vigorous star formation. We note that while the star formation is always efficient, the massive system, whose original progenitors had gas fractions of $f_{\text {gas }}=0.4$, becomes largely dissipationless after $t-t_{\text {disk }}=0.4 h^{-1} \mathrm{Gyr}$ when the total gas fraction drops below $f_{\text {gas }}=0.06$ and the amount of cold, star forming gas is a fraction $\sim 0.30$ of the gas mass. Dissimilarly, the small mass system experiences large variations in its consumption timescale, with extremely efficient star formation at the first pericentric passage $\left(t-t_{\text {disk }} \approx\right.$ $0.1-0.2 h^{-1}$ Gyr $)$ and the final coalescence $\left(t-t_{\text {disk }} \approx 0.85 h^{-1}\right.$ Gyr) and inefficient star formation at larger progenitor separations and after the merger is completed. We comment that this variation in consumption timescale for small mass systems differs importantly from the scenario described by Bekki (1998), who infers that tilt in the fundamental plane may arise from a gradation in consumption timescale or star formation "rapidity" with galaxy mass. While the time-averaged consumption timescale in smaller systems is longer as emphasized by Bekki (1998), we do not introduce an additional ad hoc mass-dependency to the gas consumption timescale. Differences in the gas consumption timescale as a function of galaxy mass in our modeling owe to the increase in disk gas density with galaxy mass, with low mass systems naturally having comparatively inefficient star formation relative to massive systems in quiescence. However, during phases of strong interaction, the gas density in low mass systems increases dramatically and leads to a rapid decrease in the gas consumption timescale. The dynamism of the consumption timescale in small mass systems then allows the less massive system to "save" its gas for strong interactions, when dissipation is very effective at converting gas into stars in the centralmost regions on a short timescale (e.g. Mihos \& Hernquist 1994).

\section{DISCUSSION}

Our simulations indicate that mergers of gas-rich disk galaxies produce tilt in the Fundamental Plane relationship relative to the scalings predicted by the virial theorem for homologous systems. The remnants of gas-rich mergers are not homologous as gas dissipation is more effective at redistributing stellar material relative to dark matter in smaller systems than in larger ones. Strongly dissipational mergers also appear necessary to steepen the $R_{\mathrm{e}}-M_{\star}$ relation of disk galaxy remnants to more closely follow the observed Shen et al. (2003) relation for early-type galaxies.

Most conservatively interpreted, our results demonstrate simply that if elliptical galaxies originate in gas-rich mergers then they will obey a tight fundamental plane relation that has significant tilt owing to dissipative effects including central $M_{\text {total }} / M_{\star}$ variations as a function of galaxy mass. However, independent observational and theoretical evidence suggests that gas-rich mergers are a necessary feature of the merger hypothesis as an elliptical galaxy formation scenario.

Bender et al. (1992) provided an early characterization of the importance of dissipation varying with the mass of elliptical galaxies. In their "gas/stellar" (GS) continuum, massive systems experience less dissipation, slowly rotate, and are increasingly flattened by anisotropy. Our work describes the impact of the GS continuum on the central structure of elliptical galaxies as a function of their stellar mass and details the origin of the GS continuum in terms of the inefficient cooling of collisionally heated gas in massive systems and dynamic variations in the gas consumption timescale in smaller mass mergers. As we note in $\$ 5.4$ these effects differ substantially in detail from previous suggestions that the gas consumption timescale was important for elliptical FP properties (e.g. Bekki 1998). Other theoretical studies of elliptical galaxy formation have suggested that gas dissipation was necessary for setting or maintaining elliptical galaxy scaling laws, notably Ciotti \& van Albada (2001) who conclude from dissipationless mergers that significant dissipation is required to simultaneously satisfy both the $M_{\mathrm{BH}}-\sigma$ and FP relation. Combined with the results of Di Matteo et al. (2005) and Robertson et al. (2005a), this work demonstrates that highly dissipational mergers can generate both the FP and $M_{\mathrm{BH}}-\sigma$ relations with tight scatter. Our analysis has also provided a detailed explanation of how dissipation systematically varies with galaxy mass and to what extent it induces FP tilt.

Furthermore, our dissipationless simulations are consistent with the emerging picture that at least one generation of spheroidal mergers do not strongly invalidate the scaling laws of their elliptical galaxy progenitors (Capelato et al. 1995; Dantas et al. 2003; Nipoti et al. 2003; Boylan-Kolchin et al. 2005). The recent observations suggesting that elliptical galaxies undergo $0.5-1$ such mergers at redshifts $z<1$ (Bell et al. 2005; van Dokkum 2005) may require such behavior as the tight local FP relation observationally limits the possible importance of scenarios that substantially increase the FP scatter. We also note here that the results from our simulations of the $M_{\mathrm{BH}}-\sigma$ relation produced by re-mergers of ellipticals are in rough agreement with previous studies (e.g. Nipoti et al. 2003). Moreover, the fact that re-merger remnants do not increase in velocity dispersion dramatically while their black hole masses double suggests that if black holes are overly massive relative to their host bulges at high redshifts (e.g.Walter et al. 2004; Peng et al. 2005), then major dissipationless mergers of spheroids will only exacerbate their discrepancy from the local $M_{\mathrm{BH}}-\sigma$ relation (Gebhardt et al. 2000; Ferrarese \& Merritt 2000; Tremaine et al. 2002). If the structural properties of quiescent galaxies instead increase the central velocity dispersions at a given black hole mass at high redshifts, major dissipationless mergers of spheroids will decrease the scatter in the $M_{\mathrm{BH}}-\sigma$ relation locally (Robertson et al. 2005b). Lastly, if the frequency of major dissipationless mergers increases with galaxy mass then the high-mass end of the $M_{\mathrm{BH}}-\sigma$ relation may steepen relative to the Tremaine et al. (2002) best-fit relation and appear as a mass-dependent scatter in the data (Robertson et al. 2005b).

Independent evidence from simulations that dissipation is important for the properties of elliptical galaxies is mounting. Using dissipational simulations of disk galaxy mergers, including a subset of the simulations analyzed in this work, Cox et al. (2005b) demonstrate that progenitor gas fractions of order $f_{\text {gas }} \approx 0.3$ or higher are necessary to reproduce the 
kinematic structure of elliptical galaxies as a function of their stellar mass. Notably, the $v / \sigma$ ratio of rotation to dispersion of remnants from $f_{\text {gas }}=0.4$ mergers better reproduces the observed distribution of elliptical kinematics than do purely dissipationless disk galaxy mergers (e.g. Naab \& Burkert 2003). Our analysis demonstrates that the Cox et al. (2005b) remnants also lie on a tight FP relation.

While recent observational determinations of the fundamental plane in galaxies at redshifts $z \sim 1$ suggest that the $M / L$ offset of the FP changes with redshift owing to passive evolution of the stellar populations, there is still disagreement regarding the redshiftevolution of the FP tilt (e.g. Gebhardt et al. 2003; van Dokkum \& Stanford 2003; van der Wel et al. 2005; Treu et al. 2005; di Serego Alighieri et al. 2005). The results of our simulations of disk galaxy mergers appropriate for higher redshifts suggest that if the $M / L$ tilt induced by stellar population effects remain fixed as a function of galaxy mass with redshift (i.e. evolution in the stellar populations only affects the FP zero-point), then the observed FP tilt will remained fixed out to high redshifts. The implication from stellar population modeling that elliptical galaxies likely formed early $(z>1-2)$ then may not provide a tight constraint on the merger hypothesis as a theory for elliptical galaxy formation since gas-rich disk galaxy models appropriate for a large range in redshift produce the same stellar-mass FP relation. As methods for including a cosmological accounting of elliptical galaxy formation times and stellar population color evolution into structure formation theory improve (see, e.g. Hopkins et al. 2005b, for a related methodology), theoretical models will have more to say in the future on this topic.

As a final note, some recent observations have been interpreted as evidence that the FP tilt is consistent with a purely stellar-population induced $M / L$ variation with galaxy mass (e.g. Cappellari et al. 2005). The success of Cox et al. (2005b) at reproducing the kinematic properties of elliptical galaxies with gas-rich disk galaxy merger simulations (including some simulations analyzed in this paper) argues strongly that dissipation is important for understanding kinematical mass trends. Our simulations suggest that a corollary of the dissipational origin of kinematic structure in ellipticals is FP tilt, which at least partially owes to central $M_{\text {total }} / M_{\star}$ variations with galaxy mass. We note that this tilt may be in addition to both kinematic nonhomology-related and photometric nonhomology-related tilt, and a full characterization of these effects is planned for future work.

\section{SUMMARY}

We determine the Fundamental Plane and $R_{\mathrm{e}}-M_{\star}$ relations produced by the merging of galaxies appropriate for redshifts $z=0-6$. We demonstrate that gas dissipation induces tilt into the Fundamental Plane, causing a deviation from the theoretical scalings derived from the virial theorem for homologous systems. Furthermore, the recovery of the $R_{\mathrm{e}}-M_{\star}$ relation for early-type galaxies similarly requires gas-rich progenitors to steepen the relation relative to the $R_{\mathrm{e}}-M_{\star}$ relation produced by the dissipationless merging of disk galaxies. These simulations argue that the merger hypothesis for the formation of locally-observed elliptical galaxies is successful if disk galaxies merge when dissipational effects are sufficiently important, requiring gas fractions $f_{\text {gas }}>0.3$ in disk galaxies at redshifts where ellipticals are primarily formed (typically $z>1$ ). We provide a detailed summary of these results below.
- The Fundamental Plane (FP) relation produced by the merging of dissipationless disk galaxy models lies nearly parallel to the plane defined by the virial relation for homologous systems. Increasing the angular momentum of the orbit by lengthening the pericentric passage distance of the orbit produces an offset in the FP by increasing the effective radius of the remnants. Scaling the disk galaxy models for redshifts $z=0-6$ produces the same FP relation. The effective radius $R_{\mathrm{e}}-$ stellar mass $M_{\star}$ relation produced in these mergers is shallower than that measured for massive galaxies in the Sloan Digital Sky Survey (Shen et al. 2003). Wide orbits and progenitors appropriate for low-redshifts produce larger remnants than do high- redshift progenitors or progenitors with bulges, but all dissipationless mergers produce shallow $R_{\mathrm{e}}-M_{\star}$ relations. We also use higher resolution simulations to demonstrate that these results are insensitive to our numerical resolution.

- The merging of gas-rich disk galaxy models with cooling, star formation, and supernova feedback produces a Fundamental Plane (FP) scaling $\left(R_{\mathrm{e}} \propto \sigma^{1.58} I_{\mathrm{e}}^{-0.80}\right)$ similar to the observed infrared FP $\left(R_{\mathrm{e}} \propto \sigma^{1.53} I_{\mathrm{e}}^{-0.79}\right.$; Pahre et al. 1998b) and is almost independent of the redshift scalings of the progenitor systems. The effective radius $R_{\mathrm{e}}-$ stellar mass $M_{\star}$ relation produced by these mergers is roughly parallel to that measured for massive galaxies in the Sloan Digital Sky Survey (Shen et al. 2003), but the chosen prograde-prograde coplanar orbit generates remnants that lie somewhat below this relation. Higher-redshift progenitors produce smaller remnants.

- The Fundamental Plane (FP) relation produced by the merging of gas-rich disk galaxies with cooling, star formation, supernova feedback, and a prescription for feedback from accreting supermassive black holes exhibits a scaling $\left(R_{\mathrm{e}} \propto \sigma^{1.55} I_{\mathrm{e}}^{-0.82}\right)$ similar to the observed infrared FP $\left(R_{\mathrm{e}} \propto \sigma^{1.53} I_{\mathrm{e}}^{-0.79} ;\right.$ Pahre et al. 1998b) and is nearly coincident with the FP produced by similar simulations without black holes. The FP relation is roughly independent of the redshift scalings of the progenitor systems and the location of remnants within the FP is fairly insensitive to a large variety of disk orientations and orbital configurations as changes in the effective radius are compensated by changes in the velocity dispersion and surface mass density. The effective radius $R_{\mathrm{e}}$ - stellar mass $M_{\star}$ relation produced by the same simulations produces a $R_{\mathrm{e}}-M_{\star}$ relation roughly parallel to that measured for massive galaxies in the Sloan Digital Sky Survey (Shen et al. 2003), with an offset toward smaller remnants. Varying the system angular momentum through combinations of the initial disk orientation and pericentric passage distance for a single pair of progenitor models produces a spread in the remnant effective radius, and a proper accounting of cosmological orbits would decrease the discrepancy with the Shen et al. (2003) normalization. The $R_{\mathrm{e}}-M_{\star}$ relation shifts toward smaller remnants as the redshift of the progenitor systems increases.

- We estimate that $\approx 40-100 \%$ of the Fundamental Plane (FP) tilt produced in the simulations owes to an increase in stellar mass at high phase-space densities and bind- 
ing energies as gas converts into stars in the centralmost regions of the remnants. This increase in phasespace density corresponds to a decrease in the totalto-stellar mass ratio $M_{\text {total }} / M_{\star}$ in the central regions of simulated elliptical galaxies. Furthermore, the decrease in $M_{\text {total }} / M_{\star}$ is a function of galaxy mass, with a stronger decrease in smaller systems, thereby inducing a FP tilt. We find that the gas-rich mergers $\left(f_{\text {gas }}>0.3\right)$ required to steepen the $R_{\mathrm{e}}-M_{\star}$ relation to become parallel with the observed Shen et al. (2003) relation also produce significant FP tilt.

- The mass-dependent impact of dissipation on the central $M_{\text {total }} / M_{\star}$ of galaxies is tied to scale-dependent processes in the merging systems. In massive halos, a substantial fraction of the available gas is collisionally heated during the first pericentric passage and driven into the halo. This heated gas cannot cool efficiently, thereby causing massive systems to act more like dissipationless systems than low mass merging galaxies. Moreover, the dynamic variation of the consumption timescale in low mass galaxies allows for the rapid conversion of gas into stars during only the most violent interactions of the merger while conserving the gas content of the system in more quiescent phases and allowing for gas dissipation to have an increased impact on the central properties of the remnants.

- The Fundamental Plane (FP) relation produced by the merging of spheroidal Hernquist (1990) galaxy models with dark matter halos remains similar to the FP delineated by their progenitors, in agreement with previous results (e.g. Boylan-Kolchin et al. 2005). Additionally, the re-merging of the remnants from gas-rich disk galaxy mergers also roughly maintains the FP of their progenitors. The effective radius $R_{\mathrm{e}}-$ stellar mass $M_{\star}$ relations produced by the merging of spheroidal galaxy models produces a slightly shallower $R_{\mathrm{e}}-M_{\star}$ relation than that delineated by the progenitor models, and although the remnants nearly double in effective radius through equal-mass mergers, a single generation of remnant re-merging will likely induce scatter but not destroy the $R_{\mathrm{e}}-M_{\star}$ correlation.

- The re-merging of ellipticals formed from the merging of disk galaxies will likely induce additional scatter in the local black hole mass $\left(M_{\mathrm{BH}}\right)-$ stellar velocity dispersion $(\sigma)$ relation, but should not destroy the correlation after a single generation of remnant remerging. In agreement with previous simulations (e.g. Nipoti et al. 2003), we find that the velocity dispersions of the re-merger remnants do not increase substantially relative to their progenitors during binary merg- ers. However, the typical number of $0.5-1$ dissipationless mergers below $z=1$ recently inferred from observations (e.g. Bell et al. 2005; van Dokkum 2005) should not dramatically change the $M_{\mathrm{BH}}-\sigma$ scalings imprinted at higher redshifts. If the velocity dispersion in remnants for a given black hole mass is larger at high redshifts than that observed locally, as inferred from simulations of the generation of the $M_{\mathrm{BH}}-\sigma$ relation from the merger of disk galaxies at redshifts $z=0-6$ (Robertson et al. 2005b), the dissipationless re-merging of remnants would decrease the scatter in the local $M_{\mathrm{BH}}-\sigma$ relationship under the condition that the strength of black hole feedback did not vary significantly with redshift. If black holes are substantially more massive than their host bulges at high redshifts (e.g. Walter et al. 2004; Peng et al. 2005), major dissipationless mergers of spheroids at low redshifts would exacerbate the discrepancy of those galaxies with the local $M_{\mathrm{BH}}-\sigma$ relation. An increasing frequency of major dissipationless mergers with galaxy mass may steepen the high-mass $M_{\mathrm{BH}}-\sigma$ relation relative to the local best-fit trend (e.g. Tremaine et al. 2002).

The success of the merger hypothesis for elliptical galaxy formation seems intimately connected with the physical process of dissipation. Our results demonstrate that if disk galaxy mergers are strongly dissipational, tilt is generated in Fundamental Plane and the $R_{\mathrm{e}}-M_{\star}$ relation steepens to approach the observed scaling. Combined with compelling evidence from the kinematic structure of simulated elliptical galaxies (e.g. Cox et al. 2005b), we conclude from our results that the formation of elliptical galaxies most likely occurred in gas-rich mergers of disk galaxies. Reconciling observational determinations of the Fundamental Plane with the merger hypothesis will require the abandonment of simplistic assumptions for the relative stellar and dark matter mass distributions at the centers of elliptical galaxies for the interpretation of observations and require more sophisticated modeling of cosmological structure formation, including a full cosmologically motivated distribution of elliptical stellar colors and metallicities.

BR acknowledges helpful conversations with Alar Toomre, Luca Ciotti, Joel Primack, Eliot Quataert, and Sandy Faber, and would like to thank Mike Boylan-Kolchin for detailed comparisons of analysis techniques. This work was supported in part by NSF grants ACI 96-19019, AST 00-71019, AST 02-06299, and AST 03-07690, and NASA ATP grants NAG5-12140, NAG5-13292, and NAG5-13381. The simulations were performed at the Center for Parallel Astrophysical Computing at Harvard-Smithsonian Center for Astrophysics.

\section{REFERENCES}

Aarseth, S. J., \& Fall, S. M. 1980, ApJ, 236, 43

Abadi, M. G., Navarro, J. F., Steinmetz, M., \& Eke, V. R. 2003, ApJ, 591, 499

Aceves, H., \& Velázquez, H. 2005, MNRAS, 360, L50

Barden, M., et al. 2005, ApJ, submitted, astro-ph/0502416

Barnes, J., \& Hut, P. 1986, Nature, 324, 446

Barnes, J. E. 1992, ApJ, 393, 484

Barnes, J. E., \& Hernquist, L. 1992, ARA\&A, 30, 705

-. 1996, ApJ, 471, 115

Barnes, J. E., \& Hernquist, L. E. 1991, ApJ, 370, L65

Bekki, K. 1998, ApJ, 496, 713
Bell, E. F., et al. 2005, ApJ, in press

Bender, R., Burstein, D., \& Faber, S. M. 1992, ApJ, 399, 462

-. 1993, ApJ, 411, 153

Bender, R., Saglia, R. P., \& Gerhard, O. E. 1994, MNRAS, 269, 785

Bender, R., Saglia, R. P., Ziegler, B., Belloni, P., Greggio, L., Hopp, U., \&

Bruzual, G. 1998, ApJ, 493, 529

Bender, R., Ziegler, B., \& Bruzual, G. 1996, ApJ, 463, L51

Bernardi, M., Renzini, A., da Costa, L. N., Wegner, G., Alonso, M. V.,

Pellegrini, P. S., Rité, C., \& Willmer, C. N. A. 1998, ApJ, 508, L143

Bernardi, M., et al. 2003a, AJ, 125, 1866

Bernardi, M., et al. 2003b, AJ, 125, 1882 
Binggeli, B., Sandage, A., \& Tarenghi, M. 1984, AJ, 89, 64

Binney, J. 1978, MNRAS, 183, 501

Binney, J., \& Tremaine, S. 1987, Galactic dynamics (Princeton, NJ, Princeton University Press, 1987, 747 p.)

Blumenthal, G. R., Faber, S. M., Flores, R., \& Primack, J. R. 1986, ApJ, 301, 27

Bondi, H. 1952, MNRAS, 112, 195

Bondi, H., \& Hoyle, F. 1944, MNRAS, 104, 273

Boylan-Kolchin, M., Ma, C.-P., \& Quataert, E. 2005, MNRAS, 362, 184

Bruzual, G., \& Charlot, S. 2003, MNRAS, 344, 1000

Bullock, J. S., Kolatt, T. S., Sigad, Y., Somerville, R. S., Kravtsov, A. V., Klypin, A. A., Primack, J. R., \& Dekel, A. 2001, MNRAS, 321, 559

Burkert, A. 1993, A\&A, 278, 23

Busarello, G., Capaccioli, M., Capozziello, S., Longo, G., \& Puddu, E. 1997, A\&A, 320, 415

Caon, N., Capaccioli, M., \& D’Onofrio, M. 1993, MNRAS, 265, 1013

Capelato, H. V., de Carvalho, R. R., \& Carlberg, R. G. 1995, ApJ, 451, 525

Cappellari, M., et al. 2005, MNRAS, in press

Ciotti, L. 1991, A\&A, 249, 99

-. 1996, ApJ, 471, 68

Ciotti, L., \& Bertin, G. 1999, A\&A, 352, 447

Ciotti, L., Lanzoni, B., \& Renzini, A. 1996, MNRAS, 282, 1

Ciotti, L., \& van Albada, T. S. 2001, ApJ, 552, L13

Cole, S., Lacey, C. G., Baugh, C. M., \& Frenk, C. S. 2000, MNRAS, 319, 168

Cox, T. J., Di Matteo, T., Hernquist, L., Hopkins, P. F., Robertson, B., \& Springel, V. 2005a, ApJ, submitted, astro-ph/0504156

Cox, T. J., Dutta, S. N., Di Matteo, T., Hernquist, L., Robertson, B., \& Springel, V. 2005b, in preparation

Cox, T. J., Primack, J., Jonsson, P., \& Somerville, R. S. 2004, ApJ, 607, L87

Dantas, C. C., Capelato, H. V., Ribeiro, A. L. B., \& de Carvalho, R. R. 2003, MNRAS, 340, 398

Davies, R. L., \& Birkinshaw, M. 1988, ApJS, 68, 409

Davies, R. L., Efstathiou, G., Fall, S. M., Illingworth, G., \& Schechter, P. L. 1983, ApJ, 266, 41

de Carvalho, R. R., \& Djorgovski, S. 1992, ApJ, 389, L49

de Vaucouleurs, G. 1948, Annales d'Astrophysique, 11, 247

-. 1961, ApJS, 5, 233

Di Matteo, T., Springel, V., \& Hernquist, L. 2005, Nature, 433, 604

di Serego Alighieri, S., et al. 2005, A\&A, 442, 125

Djorgovski, S., \& Davis, M. 1987, ApJ, 313, 59

Dressler, A. 1984, ApJ, 281, 512

Dressler, A., Lynden-Bell, D., Burstein, D., Davies, R. L., Faber, S. M., Terlevich, R., \& Wegner, G. 1987, ApJ, 313, 42

Dubinski, J. 1998, ApJ, 502, 141

Faber, S. M. 1973, ApJ, 179, 731

Faber, S. M., Dressler, A., Davies, R. L., Burstein, D., \& Lynden-Bell, D. 1987, in Nearly Normal Galaxies. From the Planck Time to the Present, 175-183

Faber, S. M., \& Jackson, R. E. 1976, ApJ, 204, 668

Fall, S. M., \& Efstathiou, G. 1980, MNRAS, 193, 189

Farouki, R. T., \& Shapiro, S. L. 1982, ApJ, 259, 103

Farouki, R. T., Shapiro, S. L., \& Duncan, M. J. 1983, ApJ, 265, 597

Ferrarese, L., \& Merritt, D. 2000, ApJ, 539, L9

Fish, R. A. 1964, ApJ, 139, 284

Franx, M. 1993, PASP, 105, 1058

Gebhardt, K., et al. 2000, ApJ, 539, L13

Gebhardt, K., et al. 2003, ApJ, 597, 239

Gerhard, O., Kronawitter, A., Saglia, R. P., \& Bender, R. 2001, AJ, 121, 1936

Gerhard, O. E. 1981, MNRAS, 197, 179

Gingold, R. A., \& Monaghan, J. J. 1977, MNRAS, 181, 375

González-García, A. C., \& van Albada, T. S. 2003, MNRAS, 342, L36

Graham, A., \& Colless, M. 1997, MNRAS, 287, 221

Guzman, R., Lucey, J. R., \& Bower, R. G. 1993, MNRAS, 265, 731

Hernquist, L. 1990, ApJ, 356, 359

-. 1992, ApJ, 400, 460

-. 1993, ApJ, 409, 548

Hernquist, L., Spergel, D. N., \& Heyl, J. S. 1993, ApJ, 416, 415

Hibbard, J. E., \& van Gorkom, J. H. 1996, AJ, 111, 655

Hopkins, P. F., Hernquist, L., Cox, T. J., Di Matteo, T., Robertson, B., \& Springel, V. 2005a, ApJS, accepted

Hopkins, P. F., Hernquist, L., Cox, T. J., Robertson, B., \& Springel, V. 2005b, ApJ, submitted, astro-ph/0508167

Hoyle, F., \& Lyttleton, R. A. 1939, in Proceedings of the Cambridge Philisophical Society, 405-+

Jørgensen, I., Franx, M., Hjorth, J., \& van Dokkum, P. G. 1999, MNRAS, 308,833

Jørgensen, I., Franx, M., \& Kjaergaard, P. 1992, A\&AS, 95, 489
-. 1993, ApJ, 411, 34

-. 1996, MNRAS, 280, 167

Kelson, D. D., Illingworth, G. D., van Dokkum, P. G., \& Franx, M. 2000a, ApJ, 531, 137

-. 2000b, ApJ, 531, 159

-. 2000c, ApJ, 531, 184

Kelson, D. D., van Dokkum, P. G., Franx, M., Illingworth, G. D., \& Fabricant, D. 1997, ApJ, 478, L13+

Kennicutt, R. C. 1998, ApJ, 498, 541

Kochanek, C. S., et al. 2000, ApJ, 543, 131

Kormendy, J. 1977, ApJ, 218, 333

Kormendy, J., \& Illingworth, G. 1982, ApJ, 256, 460

Kronawitter, A., Saglia, R. P., Gerhard, O., \& Bender, R. 2000, A\&AS, 144, 53

Lucey, J. R., Bower, R. G., \& Ellis, R. S. 1991a, MNRAS, 249, 755

Lucey, J. R., Guzman, R., Carter, D., \& Terlevich, R. J. 1991b, MNRAS, 253, 584

Lucy, L. B. 1977, AJ, 82, 1013

McIntosh, D. H., et al. 2005, ApJ, 632, 191

McKee, C. F., \& Ostriker, J. P. 1977, ApJ, 218, 148

Mihos, J. C., \& Hernquist, L. 1994, ApJ, 431, L9

- 1996, ApJ, 464, 641

Mo, H. J., Mao, S., \& White, S. D. M. 1998, MNRAS, 295, 319

Mobasher, B., Guzman, R., Aragon-Salamanca, A., \& Zepf, S. 1999, MNRAS, 304, 225

Naab, T., \& Burkert, A. 2001, ApJ, 555, L91

- 2003, ApJ, 597, 893

Naab, T., Burkert, A., \& Hernquist, L. 1999, ApJ, 523, L133

Navarro, J. F., Frenk, C. S., \& White, S. D. M. 1997, ApJ, 490, 493

Nipoti, C., Londrillo, P., \& Ciotti, L. 2003, MNRAS, 342, 501

Padmanabhan, N., et al. 2004, New Astronomy, 9, 329

Pahre, M. A., de Carvalho, R. R., \& Djorgovski, S. G. 1998a, AJ, 116, 1606

Pahre, M. A., Djorgovski, S. G., \& de Carvalho, R. R. 1995, ApJ, 453, L17+ -. 1998b, AJ, 116, 1591

Peng, C. Y., Impey, C. D., Ho, L. C., Barton, E. J., \& Rix, H.-W. 2005, ApJ, in press

Poveda, A. 1958, Boletin de los Observatorios Tonantzintla y Tacubaya, 2, 3

Prugniel, P., \& Simien, F. 1994, A\&A, 282, L1

-. 1996, A\&A, 309, 749

Ravindranath, S., et al. 2004, ApJ, 604, L9

Robertson, B., Hernquist, L., Bullock, J. S., Cox, T. J., Di Matteo, T., Springel, V., \& Yoshida, N. 2005a, ApJ, submitted, astro-ph/0503369

Robertson, B., Hernquist, L., Cox, T. J., Di Matteo, T., Hopkins, P. F., Martini, P., \& Springel, V. 2005b, ApJ, submitted, astro-ph/0506038

Robertson, B., Yoshida, N., Springel, V., \& Hernquist, L. 2004, ApJ, 606, 32

Rusin, D., et al. 2003, ApJ, 587, 143

Sáiz, A., Domínguez-Tenreiro, R., \& Serna, A. 2004, ApJ, 601, L131

Saglia, R. P., Bender, R., \& Dressler, A. 1993, A\&A, 279, 75

Sandage, A. 1972, ApJ, 176, 21

Schade, D., Barrientos, L. F., \& Lopez-Cruz, O. 1997, ApJ, 477, L17+

Schmidt, M. 1959, ApJ, 129, 243

Sersic, J. L. 1968, Atlas de galaxias australes (Cordoba, Argentina:

Observatorio Astronomico, 1968)

Shen, S., Mo, H. J., White, S. D. M., Blanton, M. R., Kauffmann, G., Voges, W., Brinkmann, J., \& Csabai, I. 2003, MNRAS, 343, 978

Siegel, M. H., Majewski, S. R., Reid, I. N., \& Thompson, I. B. 2002, ApJ, 578,151

Springel, V. 2005, MNRAS, in press

Springel, V., Di Matteo, T., \& Hernquist, L. 2005, MNRAS, 361, 776

Springel, V., \& Hernquist, L. 2002, MNRAS, 333, 649

-. 2003, MNRAS, 339, 289

-. 2005, ApJ, 622, L9

Stebbins, J., \& Whitford, A. E. 1952, ApJ, 115, 284

Terlevich, R., Davies, R. L., Faber, S. M., \& Burstein, D. 1981, MNRAS, 196, 381

Tonry, J. L., \& Davis, M. 1981, ApJ, 246, 680

Toomre, A. 1964, ApJ, 139, 1217

Toomre, A. 1977, in Evolution of Galaxies and Stellar Populations, 401-+

Toomre, A., \& Toomre, J. 1972, ApJ, 178, 623

Tremaine, S., et al. 2002, ApJ, 574, 740

Treu, T., Ellis, R. S., Liao, T. X., \& van Dokkum, P. G. 2005, ApJ, 622, L5

Treu, T., Stiavelli, M., Bertin, G., Casertano, S., \& Møller, P. 2001, MNRAS, 326, 237

Treu, T., Stiavelli, M., Casertano, S., Møller, P., \& Bertin, G. 1999, MNRAS, 308, 1037

-. 2002, ApJ, 564, L13

Trujillo, I., \& Aguerri, J. A. L. 2004, MNRAS, 355, 82

Trujillo, I., et al. 2004, ApJ, 604, 521 
van der Wel, A., Franx, M., van Dokkum, P. G., \& Rix, H.-W. 2004, ApJ, 601, L5

van der Wel, A., Franx, M., van Dokkum, P. G., Rix, H.-W., Illingworth, G. D., \& Rosati, P. 2005, ApJ, 631, 145

van Dokkum, P. G. 2005, AJ, 130, 2647

van Dokkum, P. G., \& Ellis, R. S. 2003, ApJ, 592, L53

van Dokkum, P. G., \& Franx, M. 1996, MNRAS, 281, 985

-. 2001, ApJ, 553, 90

van Dokkum, P. G., Franx, M., Kelson, D. D., \& Illingworth, G. D. 1998 , ApJ, 504, L17+

-. 2001, ApJ, 553, L39 van Dokkum, P. G., \& Stanford, S. A. 2003, ApJ, 585, 78

Vitvitska, M., Klypin, A. A., Kravtsov, A. V., Wechsler, R. H., Primack, J. R., \& Bullock, J. S. 2002, ApJ, 581, 799

Walter, F., Carilli, C., Bertoldi, F., Menten, K., Cox, P., Lo, K. Y., Fan, X., \& Strauss, M. A. 2004, ApJ, 615, L17

White, S. D. M. 1979, MNRAS, 189, 831

Woo, J., Urry, C. M., Lira, P., van der Marel, R. P., \& Maza, J. 2004, ApJ, 617, 903 